\title{
Instability of Surface Quasigeostrophic Spatially Periodic Flows
}

\author{
M. V. KALASHNIK AND M. V. KURGANSKY \\ A. M. Obukhov Institute of Atmospheric Physics, Russian Academy of Sciences, Moscow, Russia \\ S. V. KOSTRYKIN \\ G. I. Marchuk Institute of Numerical Mathematics, and A. M. Obukhov Institute of Atmospheric Physics, \\ Russian Academy of Sciences, Moscow, Russia
}

(Manuscript received 17 April 2019, in final form 16 October 2019)

\begin{abstract}
The surface quasigeostrophic (SQG) model is developed to describe the dynamics of flows with zero potential vorticity in the presence of one or two horizontal boundaries (Earth surface and tropopause). Within the framework of this model, the problems of linear and nonlinear stability of zonal spatially periodic flows are considered. To study the linear stability of flows with one boundary, two approaches are used. In the first approach, the solution is sought by decomposing into a trigonometric series, and the growth rate of the perturbations is found from the characteristic equation containing an infinite continued fraction. In the second approach, few-mode Galerkin approximations of the solution are constructed. It is shown that both approaches lead to the same dependence of the growth increment on the wavenumber of perturbations. The existence of instability with a preferred horizontal scale on the order of the wavelength of the main flow follows from this dependence. A similar result is obtained within the framework of the SQG model with two horizontal boundaries. The Galerkin method with three basis trigonometric functions is also used to study the nonlinear dynamics of perturbations, described by a system of three nonlinear differential equations similar to that describing the motion of a symmetric top in classical mechanics. An analysis of the solutions of this system shows that the exponential growth of disturbances at the linear stage is replaced by a stage of stable nonlinear oscillations (vacillations). The results of numerical integration of full nonlinear SQG equations confirm this analysis.
\end{abstract}

\section{Introduction}

In geophysical fluid dynamics, the atmosphere is traditionally represented by a layer of continuously stratified rotating Boussinesq fluid with one or two horizontal boundaries - the underlying Earth surface and the tropopause (e.g., Vallis 2006). This approach was first proposed in the classical paper by Eady (1949), devoted to the study of baroclinic instability of atmospheric flows with a constant vertical shear of zonal velocity, which made it possible to determine, in good agreement with empirical data, the growth rate and preferred scale of developing baroclinic perturbations. Quasigeostrophic flows in such a fluid layer are described by the potential vorticity (PV) transport equation. The description of the dynamics is greatly simplified for flows with zero internal PV. The velocity field of such flows is fully expressed through the

Corresponding author: Dr. Michael Kurgansky, kurgansk@ifaran.ru boundary distributions of the potential temperature (buoyancy). The description of the three-dimensional (3D) dynamics of atmospheric motions in this case is reduced to solving two-dimensional (2D) buoyancy transport equations taken at the boundaries of the atmospheric layer. The corresponding dynamic model, the surface quasigeostrophic (SQG) model, was proposed by Blumen (1978) and also in Held et al. (1995). In subsequent numerous studies, the SQG model, which occupies an intermediate position between the 2D and 3D quasigeostrophic (QG) models, was instrumental for solving important geophysical problems. Atmospheric applications included a description of the dynamics of temperature anomalies on the underlying surface and the tropopause (Juckes 1994; Harvey and Ambaum 2010; Harvey et al. 2011) and an analysis of the structure and dynamics of the surface frontal zones (Hakim et al. 2002). To describe the dynamics of the upper-ocean layer with frontal properties, the SQG model was used in Lapeyre and Klein (2006), Badin (2013) and 
McWilliams (2016). Differences in energy cascades in SQG and QG turbulence models were analyzed in Capet et al. (2008), Tulloch and Smith (2009a,b), Badin (2014) and Ragone and Badin (2016). An extensive cycle of papers was devoted to the study of the dynamics of point-like and distributed SQG vortices (Muraki and Snyder 2007; Carton 2009; Dritschel 2011; Harvey and Ambaum 2011; Harvey et al. 2011; Lim and Majda 2001; Carton et al. 2016; Reinaud et al. 2016, 2017; Badin and Barry 2018; Badin and Poulin 2018; Conti and Badin 2019).

This paper is devoted to the problem of the stability of spatially periodic SQG flows, which, to our knowledge, has not previously been considered in the literature. Spatially periodic flows are described by exact solutions of the SQG equations, with one or two horizontal boundaries. As certain geophysical prototypes of flows with a periodic or almost periodic structure, can serve observed zonal flows in the atmospheres of the giant planets, in particular on Jupiter (Vasavada and Showman 2005). Analogs of such flows can also be created in the ocean under the action of the periodically in space distributed surface wind shear stress. Another mechanism leading to spatially periodic oceanic currents is associated with the so-called zonalization (zonation), that is, the formation of a system of alternating zonal jets (Berloff et al. 2009; Galperin and Read 2019). This system of multiple jets and fronts has been discovered in the Antarctic Circumpolar Current, having predominantly zonal orientation but with some deviations from it (Maximenko et al. 2005; Sokolov and Rintoul 2007). This discovery contrasts sharply with the traditional notion that there are only three persistent zonal fronts of planetary scale in the Southern Ocean. Observational data show that multiple jets are subject to meandering, merging/diverging, and changing their intensity. This indicates on the internal dynamic instability of these finescale flows of an almost periodic spatial structure and hints that SQG equations might be instrumental for such an analysis. As a hypothesis, it cannot be ruled out that the observed persistent zonal jets on giant planets have in fact a similar finescale structure in the form of alternating multiple jets and the SQG model can also be applied to the study of stability and spatiotemporal variability of these jets.

In an attempt to explain the zonalization mechanism, it was concluded in Berloff et al. (2009) that large-scale oceanic zonal jets experience baroclinic instability, which leads to formation of the alternating meridional jets, that is, the "noodles" by terminology accepted in Berloff et al. (2009). Once the noodles emerge to finite amplitude they experience the secondary, transverse instability that sets meridional scale of the multiple zonal jets, and we argue that the SQG-model, with its neglect of the beta-effect, might be helpful in studies of such secondary instability since this model-based analysis can be equally applied to zonal and meridional currents.

The linear stability theory of a spatially periodic SQG flow with one boundary (see section 2) is in many aspects similar to the theory developed for a 2D spatially periodic incompressible fluid flow, named the Kolmogorov flow (Meshalkin and Sinai 1961). An extensive bibliography of papers devoted to the study of that famous flow is presented in Lucas and Kerswell (2014), Kim and Okamoto (2015) and Kalashnik and Kurgansky (2018). As for the Kolmogorov flow, the growth increment of linear perturbations of the spatially periodic SQG flow with one horizontal boundary is found from the characteristic equation containing an infinite continued fraction; see section 3 . The existence of instability with a preferred horizontal scale on the order of the wavelength of the main flow follows from this relation. As shown in section 4, a good approximation to the characteristic equation is given by the classical Galerkin method with basis trigonometric functions depending on the transverse coordinate. The Galerkin method also allows one to describe the main features of the nonlinear dynamics of disturbances; see section 5 . In section 6, we present the results of numerical integration of full nonlinear SQG equations, which confirm the main deductions of section 5. The problem of the stability of a spatially periodic flow for a finite-height atmospheric layer, enclosed between two horizontal boundaries is considered in section 7. The obtained results are concluded in section 8. Appendix A touches upon the effect of the momentum and buoyancy horizontal diffusion on linear stability. Details of the higher-order Galerkin approximation with five basis trigonometric functions are given in appendix B.

\section{Formulation of the problem for a semi-infinite atmosphere}

A stably stratified, rotating, and semi-infinite atmosphere $(z>0)$ with a constant buoyancy frequency $N$ and a constant Coriolis parameter $f$ is considered. Atmospheric motions with a characteristic velocity $U_{0}$, horizontal scale $D$, and Rossby number Ro $=U_{0} / f D \ll 1$ are described by the transport equation for quasigeostrophic (QG) potential vorticity $q$, taken in the Boussinesq approximation. The nondimensional form of the equation is

$$
q_{t}+J(\psi, q)=0, \quad q=\nabla^{2} \psi+\psi_{z z},
$$

where $\psi$ is the geostrophic streamfunction; $J(\psi, q)=$ $\psi_{x} q_{y}-\psi_{y} q_{x}$ is the 2D Jacobian, and $\nabla^{2} \psi=\psi_{x x}+\psi_{y y}$ the 
2D Laplacian. The $x$ and $y$ axes are assumed to be directed eastward and northward, respectively. Here, $D, H=D f / N$, $T=D / U_{0}, \Psi_{0}=U_{0} D$ are the horizontal scale, the vertical scale, the time scale, and the streamfunction scale, respectively. If $\Sigma_{0}=U_{0} N$ is the buoyancy scale, then the dimensionless horizontal components of the velocity $u$ and $v$ and the buoyancy disturbance $\sigma$ are related to the streamfunction by the relations $u=-\psi_{y}, v=\psi_{x}$, and $\sigma=\psi_{z}$.

A fundamentally important boundary condition is added to (1):

$$
z=0: \quad \psi_{z t}+J\left(\psi, \psi_{z}\right)=0
$$

Equation (2) follows from the impermeability condition on the rigid horizontal boundary $z=0$ and the expression for the vertical velocity in terms of the horizontal transport of buoyancy, in the QG approximation. At the upper boundary of the atmosphere, $z \rightarrow \infty$, the solution regularity is required.

Flows with identically zero potential vorticity, $q \equiv 0$, exactly satisfy Eq. (1). The dynamics of these flows, which are similar to potential flows in classical hydrodynamics, is described by the Laplace equation solutions

$$
\psi_{x x}+\psi_{y y}+\psi_{z z}=0
$$

with nonlinear and nonstationary boundary condition (2). From (2), (3) follow the energy conservation law,

$$
d E / d t=0, \quad E=\frac{1}{2} \int_{0}^{\infty} \overline{\left(\psi_{x}^{2}+\psi_{y}^{2}+\psi_{z}^{2}\right)} d z,
$$

and the surface potential energy conservation law,

$$
d V / d t=0, \quad V=(1 / 2) \overline{\psi_{z}^{2}},
$$

where the overbar in (4a) and (4b) denotes averaging over horizontal coordinates. This is verified by direct calculus. Equations (2) and (3) underlie the SQG model with one boundary (Held et al. 1995).

Exact stationary solution of (2) and (3),

$$
\bar{\psi}=e^{-z} \cos y, \quad \bar{u}=e^{-z} \sin y, \quad \bar{\sigma}=-e^{-z} \cos y,
$$

describes a zonal spatially periodic flow localized near the underlying surface. The dimensional form of the velocity profile reads $\bar{u}=U_{0} e^{-z / H} \sin (y / D)$, where $H=$ $D f / N$. Representing $\psi=\bar{\psi}+\psi^{\prime}$ and omitting the prime over perturbations, the Laplace equation, (3), is obtained with the boundary condition

$$
z=0: \quad \psi_{z t}+\sin y\left(\psi_{x z}+\psi_{x}\right)+J\left(\psi, \psi_{z}\right)=0
$$

and the condition of regularity at $z \rightarrow \infty$. Within the framework of problem (3) and (6), linear and nonlinear dynamics of perturbations superimposed on the spatially periodic flow (5) will be studied further on. We emphasize that the nonlinear term of the problem is contained only in the boundary condition (6) and describes nonlinear advection of the surface buoyancy field.

Note that in the framework of the SQG model with two boundaries, which will be discussed in section 7 , many problems in the theory of baroclinic instability, in particular the classical Eady (1949) problem of the constant-vertical-shear flow instability (cf. Pedlosky 1987), are investigated. This circumstance, however, is usually not specially emphasized in the literature.

\section{Linear stability theory; continued fractions}

The linear stability theory of flow, (5), is in many aspects similar to the theory of Meshalkin and Sinai (1961), developed for a 2D spatially periodic flow of a viscous incompressible fluid of constant density (the so-called Kolmogorov flow) and based on the use of the theory of continued fractions. We will consider sinusoidal in coordinate $x$ perturbations with a spatial period of $2 \pi / k$ that have a spatial period of $2 \pi$ along the transverse coordinate $y$ (the period of the background flow). Such perturbations satisfy the Laplace equation, (3), and can be represented as a Fourier series:

$$
\psi=e^{\lambda t} e^{i k x} \sum_{n=-\infty}^{n=+\infty} e^{-k_{n} z} e^{i n y} \psi_{n}, \quad k_{n}=\sqrt{k^{2}+n^{2}}
$$

where $\lambda$ is the increment of perturbation growth. Representing siny in the complex form and substituting (7) into the linearized form of the boundary condition (6), we obtain an infinite system of equations for the coefficients of Fourier series:

$$
2 \lambda\left(k_{n} / k\right) \psi_{n}+\left(k_{n-1}-1\right) \psi_{n-1}-\left(k_{n+1}-1\right) \psi_{n+1}=0 .
$$

Denoting $d_{n}=\left(k_{n}-1\right) \psi_{n}$, the system (8) can be written in the form of a trinomial relation:

$$
\tilde{a}_{n} d_{n}+d_{n-1}-d_{n+1}=0
$$

with coefficients $\tilde{a}_{n}=(2 / k)\left[\lambda k_{n} /\left(k_{n}-1\right)\right]$ depending on the increment $\lambda$. As in Meshalkin and Sinai (1961), where a system similar to (9) arises, this increment is found from the characteristic equation,

$$
-\left(\tilde{a}_{0} / 2\right)=\left[0 ; \tilde{a}_{1}, \tilde{a}_{2}, \ldots\right]
$$

where, using the notations adopted in Khinchin (1964), the square brackets indicate an infinite continued fraction 


$$
\left[\tilde{b}_{0} ; \tilde{b}_{1}, \tilde{b}_{2}, \ldots\right]=\tilde{b}_{0}+\frac{1}{\tilde{b}_{1}+\frac{1}{\tilde{b}_{2}+\frac{1}{\cdots}}}
$$

Equation (10) is obtained from (9) as follows. First, by introducing $\rho_{n}=d_{n-1} / d_{n}$ we have from (9) a recurrence relation $\rho_{n}=-\tilde{a}_{n}+1 / \rho_{n+1}$, or identically $-\rho_{n}=\tilde{a}_{n}+$ $1 /\left(-\rho_{n+1}\right)$, and therefore $-\rho_{0}=\left[\tilde{a}_{0} ; \tilde{a}_{1}, \tilde{a}_{2}, \ldots\right]$. Second, introducing $\rho_{n+1}^{*}=d_{n+1} / d_{n}$ we get from (9) that $\rho_{n+1}^{*}=\tilde{a}_{n}+1 / \rho_{n}^{*}$ and therefore $\rho_{1}^{*}=\left[\tilde{a}_{0} ; \tilde{a}_{-1}, \tilde{a}_{-2}, \ldots\right] \equiv$ $\left[\tilde{a}_{0} ; \tilde{a}_{1}, \tilde{a}_{2}, \ldots\right]$. Third, by definition $\tilde{a}_{0}+\rho_{0}=\rho_{1}^{*}$, and denoting $S=\left[0 ; \tilde{a}_{1}, \tilde{a}_{2}, \ldots\right]$ we obtain that $\tilde{a}_{0}-\tilde{a}_{0}-S=$ $\tilde{a}_{0}+S$; therefore, after cancelation of similar terms we have (10). According to Khinchin (1964), for the continued fraction in the right-hand side of (10) to converge, it is necessary and sufficient that the series $\sum_{n=1}^{\infty} \tilde{a}_{n}$ diverge, which is true in our case since the common term $\tilde{a}_{n} \cong 2 \lambda / k=$ const for sufficiently large $n$.

Approximate values of $\lambda$ can be obtained by replacing the infinite continued fraction $S$ with its convergent, that is, truncating a continued fraction in (10) on the $\mathcal{N}$ th term. The one-term (first) approximation to (10), when the right-hand side is replaced by $\left[0 ; \tilde{a}_{1}\right]$, reduces to an equation $-\left(\tilde{a}_{0} / 2\right)=1 / \tilde{a}_{1}$. Now, taking into account expressions for $\tilde{a}_{0}$ and $\tilde{a}_{1}$, we get

$$
\lambda^{2}=\frac{k(1-k)\left(k_{1}-1\right)}{2 k_{1}}, \quad k_{1}=\sqrt{k^{2}+1} .
$$

Equation (11) determines the dependence of the increment $\lambda$ on the wavenumber $k$. According to (11), instability occurs when $0<k<1$, with the fastest growing perturbation corresponding to $k=k_{m} \sim 0.7$ (Fig. 1).

Truncating the infinite continued fraction $S$ on the second term, we obtain the equation $-\left(\tilde{a}_{0} / 2\right)=$ $\left[0 ; \tilde{a}_{1}, \tilde{a}_{2}\right]$, wherefore $\left(k_{2}=\sqrt{k^{2}+4}\right)$

$$
\lambda^{2}=\frac{\left(k_{1}-1\right) k\left[2 k_{2}-\left(3 k_{2}-1\right) k\right]}{4 k_{1} k_{2}} .
$$

The dependence $\lambda=\lambda(k)$ described by (12) is shown in Fig. 1 with a dashed line. Using the properties of continued fractions, it can be shown that $\left[0 ; \tilde{a}_{1}\right]<S<\left[0 ; \tilde{a}_{1}, \tilde{a}_{2}\right]$ and the exact dependence $\lambda=\lambda(k)$ lies between the curves given by (11) and (12). For a qualitative and fairly accurate quantitative analysis, we can restrict ourselves to the first approximation, (11).

However, a question may arise as how well the exact solution $\left\{d_{n}\right\}$ of (9) can be approximated when applying the procedure that leads to (11). To answer this question, we consider the effect of the momentum and buoyancy horizontal diffusion on linear

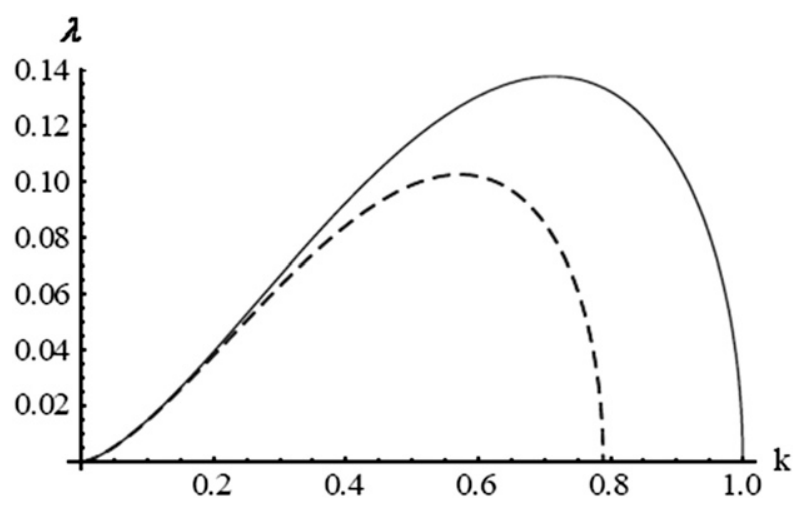

FIG. 1. Two approximations of the theory of continued fractions for the dependence of the instability increment $\lambda$ on the wavenumber $k$. The first approximation is shown by a solid line and the second approximation is shown by a dashed line. The exact dependence $\lambda(k)$ lies between these two curves.

stability of the solution (5). Details are provided in appendix A.

\section{Linear stability theory: Galerkin method}

An alternative (approximate) approach to describe the linear dynamics of perturbations is to use the Galerkin method with basis trigonometric functions of the transverse coordinate $y$. To obtain the first approximation, we take three basis functions, $f_{1}=\sin y$, $f_{2}=\cos y$, and $f_{3}=1$, and seek a solution in the form of expansion

$$
\psi=A(x, z, t) \sin y+B(x, z, t) \cos y+C(x, z, t) .
$$

By virtue of the Laplace equation, (3), the expansion coefficients satisfy the equations

$A_{x x}+A_{z z}-A=0, \quad B_{x x}+B_{z z}-B=0, \quad C_{x x}+C_{z z}=0$.

From the linearized form of condition (6), the equations relating the functions $A, B$, and $C$ on the boundary also follow. To obtain these equations, we write condition (6) in the form $z=0: \quad L(\psi)=\psi_{z t}+\sin y\left(\psi_{x z}+\psi_{x}\right)=0$ and use the orthogonality conditions $\left\langle f_{i}(x) \hat{L}(\psi)\right\rangle=0, i=$ $1,2,3$. Hereinafter, angle brackets denote the averaging operator over the spatial period $2 \pi:\langle\varphi\rangle=(2 \pi)^{-1} \int_{0}^{2 \pi} \varphi d y$. For the function (13), direct calculation gives

$$
\begin{aligned}
L(\psi)= & {\left[C_{z t}+(1 / 2)\left(A_{x}+A_{x z}\right)\right]+\left(A_{z t}+C_{x}+C_{x z}\right) \sin y } \\
& +B_{z t} \cos y+(1 / 2)\left(B_{x}+B_{x z}\right) \sin 2 y
\end{aligned}
$$

Substitution of this expression into the conditions of orthogonality leads to the equations 


$$
\begin{aligned}
& z=0: \quad A_{z t}+C_{x z}+C_{x}=0, \\
& C_{z t}+(1 / 2)\left(A_{x z}+A_{x}\right)=0, \quad B_{z t}=0 .
\end{aligned}
$$

Thus, the description of the linear dynamics of perturbations reduces to the solution of system (14) with boundary conditions (16).

Equations (14) are satisfied by harmonic functions with respect to the coordinate $x$ :

$$
A=a(t) e^{-k_{1} z} \sin k x, \quad C=c(t) e^{-k z} \cos k x, \quad B=0,
$$

where $k_{1}=\sqrt{k^{2}+1}$. Substituting (17) into (16) leads to a system of ordinary differential equations,

$$
\begin{aligned}
a_{t}+\alpha c & =0, \quad c_{t}+\gamma a=0, \quad \alpha=k(1-k) / k_{1}, \\
\gamma & =\left(k_{1}-1\right) / 2,
\end{aligned}
$$

which has solutions that grow exponentially with time, $a, c \propto e^{\lambda t}$. It is easy to verify that the increment $\lambda$ is determined by the expression (11). Thus, the Galerkin method with three basis functions leads to the same result as the first approximation in the theory of continued fractions.

A more accurate Galerkin approximation can be obtained by representing the solution in the form containing five basis functions:

$$
\begin{aligned}
\psi= & A^{(1)}(x, z, t) \sin y+B^{(1)}(x, z, t) \cos y+A^{(2)}(x, z, t) \sin 2 y \\
& +B^{(2)}(x, z, t) \cos 2 y+C(x, z, t) .
\end{aligned}
$$

It is shown in appendix B that the resulting system of five equations for the expansion coefficients splits into two subsystems, one of which describes the instability with the growth rate of perturbations (12).

\section{Nonlinear dynamics of perturbations}

By virtue of the energy conservation law, (4a), the exponential growth of linear perturbations should ultimately be replaced by a regime with finite amplitude. The description of such a regime is reduced to solving a problem with a nonlinear boundary condition, (6). An approximate solution to this nonlinear problem can also be constructed using the Galerkin method.

As before, we seek an approximate solution in the form of an expansion, (13), where the expansion coefficients satisfy linear equations (14), which follow from the Laplace equation, (3). To obtain nonlinear equations on the boundary, we transform condition (6). The linear part of this condition, $L(\psi)$, is given by expression (15). Direct calculation of the nonlinear term yields

$$
\begin{aligned}
J\left(\psi, \psi_{z}\right)= & (1 / 2)\left(B A_{z}-A B_{z}\right)_{x}+\left(B C_{x z}-C_{x} B_{z}\right) \sin y \\
& +\left(C_{x} A_{z}-A C_{x z}\right) \cos y+F(\sin 2 y, \cos 2 y),
\end{aligned}
$$

where $F(\sin 2 y, \cos 2 y)$ denotes a linear combination of trigonometric functions of double argument. Substitution of (15) and this expression into (6), with the subsequent use of the orthogonality conditions, leads to a nonlinear system of equations at the boundary $z=0$ :

$$
\begin{aligned}
A_{z t}+(B+1) C_{x z}+\left(1-B_{z}\right) C_{x} & =0, \\
C_{z t}+(1 / 2)\left[(B+1) A_{z}+\left(1-B_{z}\right) A\right]_{x} & =0, \\
B_{z t}+C_{x} A_{z}-A C_{x z} & =0 .
\end{aligned}
$$

The linearized version of (19) and (20) obviously reduces to (16).

To build solutions of a nonlinear problem that are periodic in $x$, we also use the Galerkin method. We seek approximate solutions of (14), (19), and (20) of the form

$A=a(t) e^{-k_{1} z} \sin k x, \quad C=c(t) e^{-k z} \cos k x, \quad B=b(t) e^{-z}$.

For the chosen solutions, (14) are exactly satisfied, and the boundary conditions (19) reduce to nonlinear ordinary differential equations without any approximation. This is not the case for (20). Therefore, we introduce the averaging operation over the horizontal coordinate, $\bar{\varphi}^{x}=(1 / L) \int_{0}^{L} \varphi d x, L=2 \pi / k$, and for the chosen solution calculate the averaged nonlinear term of (20): $\overline{\left(C_{x} A_{z}-A C_{x z}\right)^{x}}=(1 / 2) k\left(k_{1}-k\right) a c$. It follows from the last expression that the approximate solution for the coefficient $B$ should depend only on time and the vertical coordinate, which is taken into account in (21). Denoting $\tilde{b}=b+1$, we thus obtain a system of ordinary differential equations for describing the nonlinear dynamics of perturbations:

$$
a_{t}+\alpha \tilde{b} c=0, \quad c_{t}+\gamma \tilde{b} a=0, \quad \tilde{b}_{t}-\beta a c=0 .
$$

Coefficients of (22),

$$
\alpha=k(1-k) / k_{1}, \quad \gamma=\left(k_{1}-1\right) / 2, \quad \beta=k\left(k_{1}-k\right) / 2,
$$

depend on the wavenumber $k$ of the perturbation. For an interval of unstable wavenumbers of the linear theory, $0<k<1$, one has that $\alpha, \beta, \gamma>0$. The linearized version $(\tilde{b} \approx 1)$ of $(22)$ reduces to $(18)$.

Nonlinear equations (22) are similar to the equations describing the motion of a symmetric top in classical mechanics or of an ideal fluid in an ellipsoidal cavity. The relevant theory is detailed in Gledzer et al. (1981). 
Formally mathematically, the system in (22) with three unknowns $(a, \tilde{b}, c)$ possesses two steady solutions describing stable equilibrium states ("permanent rotations" in classical mechanics), $(a, 0,0),(0,0, c)$, and the third steady solution corresponds to the unstable equilibrium state, $(0, \tilde{b}, 0)$. An important role is played by the conservation laws arising from (22):

$$
\gamma a^{2}-\alpha c^{2}=I_{1}=\text { const }, \quad \beta a^{2}+\alpha \tilde{b}^{2}=I_{2}=\text { const } .
$$

It follows directly from the second conservation law that, as the time grows, all solutions are limited in magnitude, the latter being in contrast to the linear problem.

In relation to the problem of stability of a spatially periodic flow under consideration, the following formulations of the initial problem for (22) have physical meaning: (i) $a(0)=0, b(0)=0, c(0)=c_{0}$, that is, $a(0)=0, \quad \tilde{b}(0)=1, \quad c(0)=c_{0}$, or (ii) $a(0)=a_{0}, b(0)=0$, $c(0)=0$, that is, $a(0)=a_{0}, \quad \tilde{b}(0)=1, \quad c(0)=0$, where $a_{0}$ and $c_{0}$ are the amplitudes of initial perturbations. For all such problems, the solutions of (22) are represented in terms of elliptic functions. The corresponding solutions describe nonlinear oscillations with a period depending on the initial perturbation amplitude (nonisochronous oscillations). The linear stage of the exponential growth of disturbances is thus replaced by the stage of stable undamped oscillations.

As an example, we determine the period $T$ of oscillations for the initial problem with conditions $a(0)=0, \quad \tilde{b}(0)=1, \quad c(0)=c_{0}$. The values $I_{1}=-\alpha c_{0}^{2}$, $I_{2}=\alpha$ of the integrals (24) correspond to these conditions. Expressing from (24) $c, \tilde{b}$ through $a$ and substituting into the first equation, (22), we get

$$
a_{t}= \pm \sqrt{\beta \gamma} \sqrt{\left(x_{0}^{2}-a^{2}\right)\left(y_{0}^{2}+a^{2}\right)}
$$

where the notations $x_{0}^{2}=\alpha / \beta$ and $y_{0}^{2}=c_{0}^{2} \alpha / \gamma$ are introduced. After the separation of variables, the formula for the oscillation period $T$ follows from (25),

$$
\frac{T}{2}=\frac{1}{\sqrt{\beta \gamma}} \int_{-x_{0}}^{x_{0}} \frac{d a}{\sqrt{\left(x_{0}^{2}-a^{2}\right)\left(y_{0}^{2}+a^{2}\right)}} .
$$

Standard substitution $a=-x_{0} \cos \theta$ and elementary transformations allow writing (26) in the form

$$
\begin{aligned}
T & =(4 / n) K(m), \quad n^{2}=\alpha\left(\gamma+\beta c_{0}^{2}\right), \\
m^{2} & =\left[1+(\beta / \gamma) c_{0}^{2}\right]^{-1},
\end{aligned}
$$

where $K(m)=\int_{0}^{\pi / 2} d \theta / \sqrt{1-m^{2} \sin ^{2} \theta}$ is a complete elliptic integral of the first kind. Dependency graphs $K(m)$ are presented in Abramowitz and Stegun (1964); in particular, when $m \rightarrow 1$, then $K(m) \rightarrow \infty$; whereas, when $m \rightarrow 0$, then $K(m) \rightarrow \pi / 2$. Since $K(m)$ is an increasing function of its argument, it follows from (27) that the smaller the amplitude $c_{0}$ of the initial perturbation is, the longer is the oscillation period $T$ and, accordingly, the longer is the stage of exponential growth of linear perturbations.

The results of numerical integration of (22) for $c_{0}=$ 0.01 are presented in Fig. 2. As can be seen, for small initial amplitudes, the oscillations are clearly anharmonic in nature. While the oscillations of $a(t)$ and $\tilde{b}(t)$ occur with changing of sign, the function $c(t)$ is always nonnegative and oscillates around an average value $c_{*}>0$. The period of oscillations of $c(t)$ is equal to half the period $T$ of oscillations of $a(t)$. This follows directly from an expression $c^{2}=\alpha^{-1}\left(\gamma a^{2}-I_{1}\right)$ representing another form of the first conservation law, (24).

The results of similar calculations for $c_{0}=0.5$ are shown in Fig. 3. An increase in $c_{0}$ led to decrease in the period $T$ of oscillation, which now become similar to harmonic oscillations. Note that such oscillations are often called the supernonlinear oscillations (Kuznetsov et al. 2002).

We present numerical estimates of the oscillation period $\mathcal{T}$ (days) for a flow with velocity amplitude $U_{0}=$ $10 \mathrm{~m} \mathrm{~s}^{-1}$ and horizontal scale $D=300 \mathrm{~km}$. Now, the Rossby number equals Ro $=U_{0} / f L=0.33$ and the advective time scale is $T_{0}=D / U_{0} \approx 8.3 \mathrm{~h}$. For the initial amplitude $c_{0}=0.01$ and for $k=0.5$ the dimensionless period of oscillations, (27), equals $T \approx 204$.0. The corresponding values of the dimensional period and the half period of oscillations are $\mathcal{T} \approx 70.6$ days and $\mathcal{T} / 2 \approx 35.3$ days. For the initial amplitude $c_{0}=0.5$, the dimensionless period is $T \approx 53.67$ and the values of the dimensional period and the half period of oscillations are $\mathcal{T} \approx 20.6$ days and $\mathcal{T} / 2 \approx 10.3$ days.

Using numerical calculations, it is possible to construct shadow patterns of the full streamfunction $\psi_{*}=\bar{\psi}+\psi^{\prime}$ at different instants of time:

$$
\begin{aligned}
\psi_{*}= & {[b(t)+1] e^{-z} \cos y+a(t) e^{-k_{1} z} \sin k x \sin y } \\
& +c(t) e^{-k z} \cos k x
\end{aligned}
$$

The corresponding patterns are repeated with a period $T$; at half period $T_{1}=T / 2$, a repetition occurs with a change in the streamfunction sign. As an example, Fig. 4 shows the streamlines at the dimensionless time instants $t=30, t=40$, and $t=50$ for the parameter values $c_{0}=$ 0.01 and $k=0.5$. As can be seen, during the development of instability a system of isolated vortex structures is formed within a spatially periodic flow. This is most pronounced at the time instant $t=50$, 

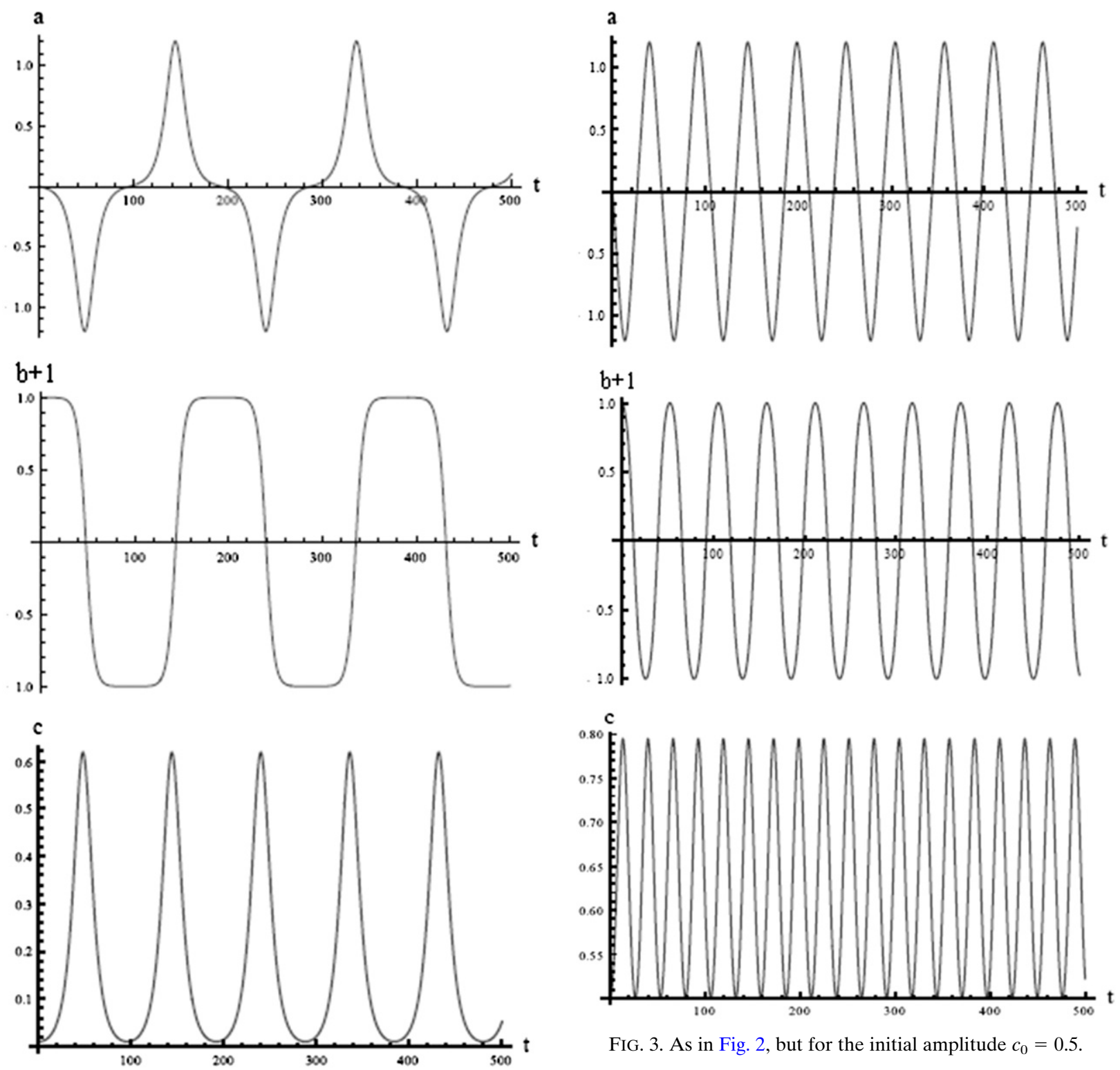

FIG. 3. As in Fig. 2, but for the initial amplitude $c_{0}=0.5$.

FIG. 2. Numerical solution of the nonlinear system (22) with initial conditions $a(0)=0, b(0)=0$, and $c(0)=c_{0}=0.01$. The disturbance wavenumber is $k=0.5$.

corresponding to half of the period $T_{1} \approx 100$. At this time instant, a periodic ensemble of closed vortices is formed, separated by narrow meandering currents, mainly directed along the meridian. The direction of the flows is explained by the fact that the time average of the streamfunction, (28), corresponds to a strictly meridional flow with streamfunction $c_{*} e^{-k z} \cos k x$.

It is important to emphasize that the approximate solution, (28), exactly satisfies the energy conservation laws, (4a) and (4b). Indeed, direct calculation of energies (squaring, integration, averaging) for solution (28) gives

$$
\begin{aligned}
V= & (1 / 2) \overline{\psi_{z}^{2}}=(1 / 4)\left[\tilde{b}^{2}+k^{2} c^{2}+(1 / 2) k_{1}^{2} a^{2}\right], \\
E= & (1 / 2) \int_{0}^{\infty} \overline{\left(\psi_{x}^{2}+\psi_{y}^{2}+\psi_{z}^{2}\right)} d z=(1 / 4)\left[\tilde{b}^{2}+k c^{2}\right. \\
& \left.+(1 / 2) k_{1} a^{2}\right] .
\end{aligned}
$$

(asterisk omitted). Taking into account the expressions for the coefficients, (23), and the first integrals, (24), it is directly verified that

$$
V=\left(I_{2}-k^{2} I_{1}\right) / 2 \alpha, \quad E=\left(I_{2}-k I_{1}\right) / 2 \alpha .
$$

Since an arbitrary function of independent integrals $I_{1}$ and $I_{2}$ is also the first integral, conservation of energies $V$, and $E$ follows from (29), as well as the physical 

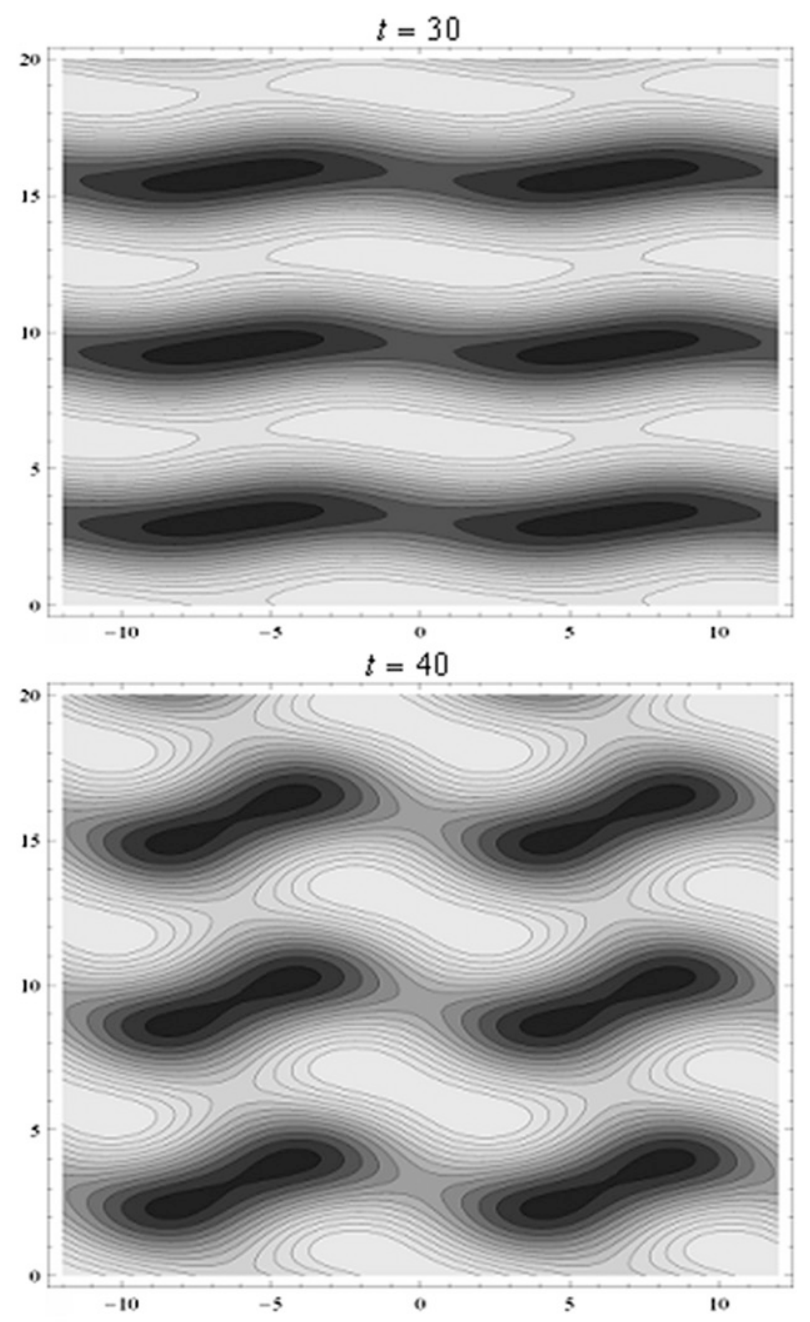

$t=50$

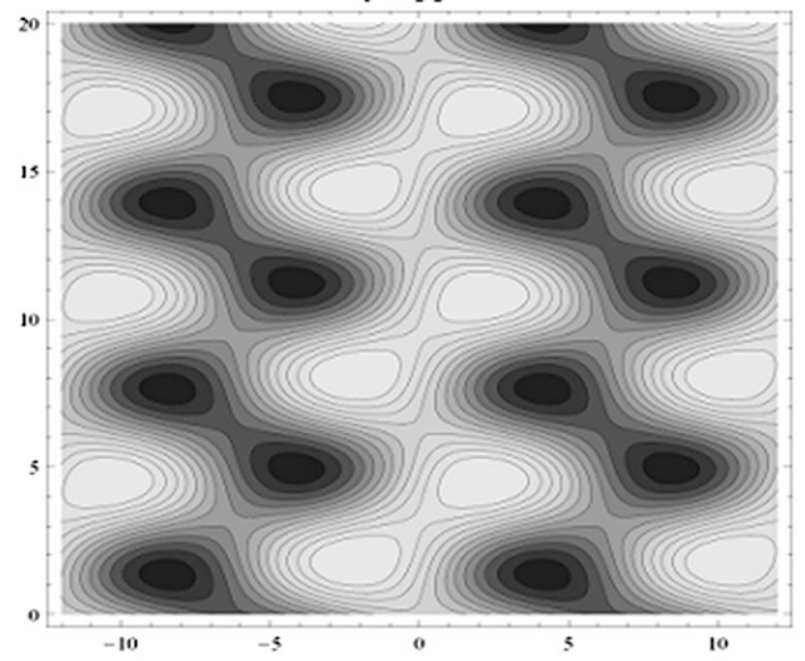

FIG. 4. Shadow patterns of the streamfunction isolines at the level $z=0$ for dimensionless time instants (top) $t=30$, (middle) $t=40$, and (bottom) $t=50$. Parameters values are $c_{0}=0.01$ and $k=0.5$. meaning of the integrals $I_{1}$ and $I_{2}$. These integrals, or more precisely their linear combinations, reflect the laws of conservation of surface potential energy and total energy.

The occurrence of oscillations is the most important feature of the nonlinear dynamics of perturbations of a spatially periodic flow. This feature reflects the fulfillment of the energy conservation law in a conservative system; apparently, it is not related to the specific structure of the flow. In this regard, the nonlinear oscillatory regime of flow instability in a two-layer quasigeostrophic model was studied in Pedlosky $(1970,1987)$, where, in particular, a colorful description is given of the mechanism of the onset of oscillations in terms of a time-periodic energy exchange between an unstable baroclinic wave and the background flow. A formula analogous to (27) for the oscillation period (in terms of an elliptic integral) is obtained by Merilees (1972).

Let us trace the fate of nonlinear oscillations in a slightly dissipative case. Following considerations of appendix A, we adopt the horizontal diffusivity value $\chi \sim 10^{4} \mathrm{~m}^{2} \mathrm{~s}^{-1}$, as a compromise, and get for the aboveindicated parameter values that the Peclet number, $\mathrm{Pe}=U_{0} D / \chi \sim 3 \times 10^{2}$. Therefore, the direct effect of dissipation on linear instability is negligible but the dissipation can affect nonlinear oscillations in the long run (cf. Ohkitani and Sakajo 2010), namely, in our case on a dissipative damping time scale $\sim D^{2} / \chi \sim 10^{7} \mathrm{~s} \sim$ 120 days, that is about six periods of oscillations, if a Cauchy-like problem with initial value $c_{0}=0.5$ is considered. Alternatively, on the attractor of dissipative system described by equations of appendix A, a sort of compensation of the effects of nonlinear instability and dissipation can occur, resulting in (almost) periodic oscillations (orbits), and nonlinear oscillations (vacillations) investigated in this section could serve as approximations for these orbits, as an anonymous reviewer suggested it. Such vacillations are indeed observed in such (slightly) dissipative systems as laboratory analogs of the atmosphere (Buzyna et al. 1989; Solomon et al. 1993).

\section{Numerical integration of full SQG equations with one boundary}

In the previous sections, the solution for the streamfunction was searched within the class of functions doubly periodic in a rectangle with the sides $L_{x}=2 \pi / k$ and $L_{y}=2 \pi$. The approximate solution, (28), is the result of expansion into a system of three long-wave Fourier harmonics, $F_{1}=\cos y, F_{2}=\sin k x \sin y$, and $F_{3}=\cos k x$. The full solution for the streamfunction should obviously be represented in the form of a doubly 

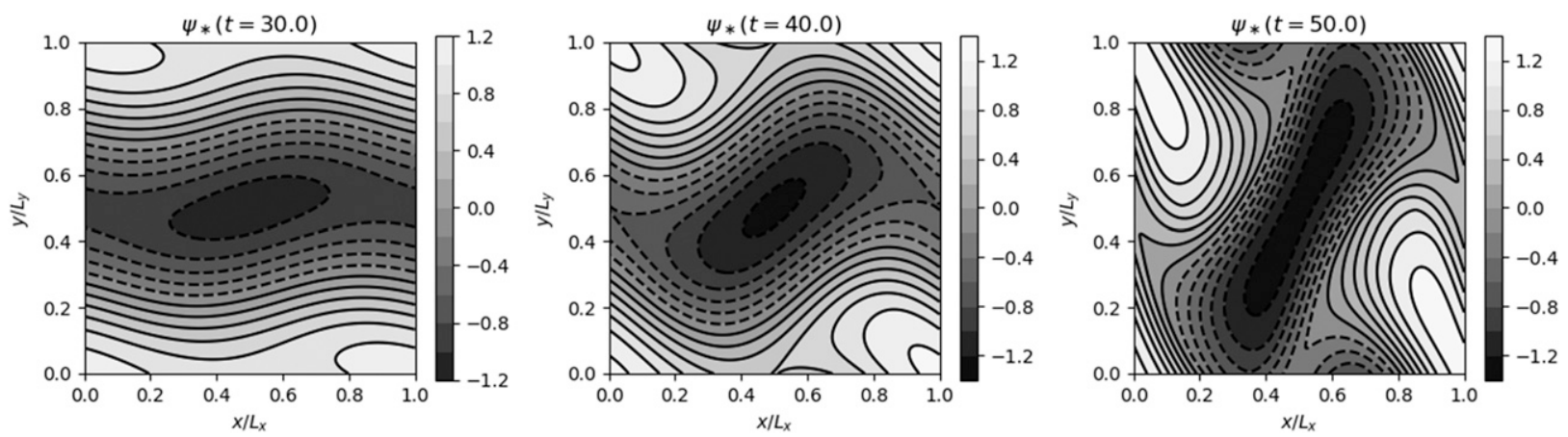

FIG. 5. The streamfunction isolines at the level $z=0$ for dimensionless time instants (left) $t=30$, (center) $t=40$, and (right) $t=50$ from numerical simulation with SQG-model for the parameters values $c_{0}=0.01, k=0.5, L_{x}=4 \pi$, and $L_{y}=2 \pi$.

periodic trigonometric series, as an infinite expansion into the Fourier harmonics with multiple periods. In this connection, the question arises: is the time-periodic behavior of the approximate solution, (28), preserved when taking into account the higher small-scale harmonics?

To address this question, we performed numerical integration of (2). Since for the solutions of Laplace equation, (3), the value of $\psi$ on boundary is expressed in terms of the normal derivative $\psi_{z}$ by means of a Hilbert-type nonlocal operator, (2) is a prognostic nonlinear equation for the buoyancy $\sigma=\psi_{z}$. This equation was solved with the initial condition $\left.\sigma\right|_{t=0}=-\cos y-k c_{0} \cos k x$; that is, the background buoyancy distribution plus a superimposed buoyancy perturbation were set at the initial time instant.

For numerical calculations we use the SQG pseudospectral code from the PyQG package (https:// pyqg.readthedocs.io/en/latest/index.html). The used pseudospectral method (Canuto et al. 1988) is based on the integration over time of a system of ordinary differential equations in spectral space, while the nonlinear terms in these equations are first calculated in physical space and only then projected into spectral space. For integration over time, the third-order Adams-Bashford scheme is used. The numerical code has an exponential filter for suppressing short-wave noise, similar to that described by Arbic and Flierl (2003).

First of all, numerical calculations confirmed the results of linear theory predicting the long-wave nature of the developing instability. In our numerical calculations, perturbations with a wavenumber $0<k<1$ increased, almost exponentially at the initial stage. Short-wave perturbations with $k>1$ were damped over time. As an example of the developing instability, Fig. 5 shows the total surface streamfunction for three dimensionless time instants $t=30, t=40$, and $t=50$ and for parameter values, $c_{0}=0.01$ and $k=0.5$. The corresponding patterns are presented in a square box, which corresponds to the transition to variables, $x^{\prime}=x / L_{x}$ and $y^{\prime}=y / L_{y}$. As in Fig. 4 constructed using the analytical solution, (28), a system of closed vortex cells is formed during the development of instability. Theoretical patterns, rearranged for a square box, are in good agreement with the patterns obtained using a numerical code.

To study the nonstationary dynamics at large times, we considered the time-dependence of the streamfunction at selected fixed points. An example of such a dependence for parameter values $c_{0}=0.5$ and $k=0.5$ and for a point $(1.2 \pi, 0.6 \pi)$ with nontransformed coordinates $(x, y)$ is presented in Fig. 6a. As it can be seen, an almost periodic oscillatory regime is established over time, that is, the regime of nonlinear vacillations. The regime remains the same for other points. The frequency spectrum in Fig. 6b shows how many time harmonics are actually involved in the formation of this regime, but still the long-frequency harmonics make the main contribution.

For a more detailed assessment of the contribution of various spatial Fourier components to the formation of the spectrum, we calculated the time dependences of the real part of their amplitudes $\tilde{\psi}_{k l}$ for each spatial harmonic (Fig. 7). The latter is characterized by a set of wavenumbers $(k, l)$, where $k$ and $l$ are the zonal and meridional wavenumbers, respectively. At $k=0.5$, the harmonics $F_{1}, F_{2}$, and $F_{3}$ correspond, respectively, to the sets $(0,1),(0.5,1)$, and $(0.5,0)$. As calculations showed, the amplitudes of the fundamental harmonics $F_{1}, F_{2}$, and $F_{3}$ are significantly superior to other amplitudes. An exception is the harmonic $(1,1)$, which also makes a significant, though somewhat lesser, contribution to the formation of the spectrum (not shown). The last harmonic, $\sin x \sin y$, that had been superimposed on the buoyancy profile, $\cos y$, in setting the initial conditions for the numerical experiments in Constantin et al. (1994) and Held et al. (1995), has, most likely, appeared to be ultimately responsible for the formation of a singular front occurring there in a rather short finite time. 

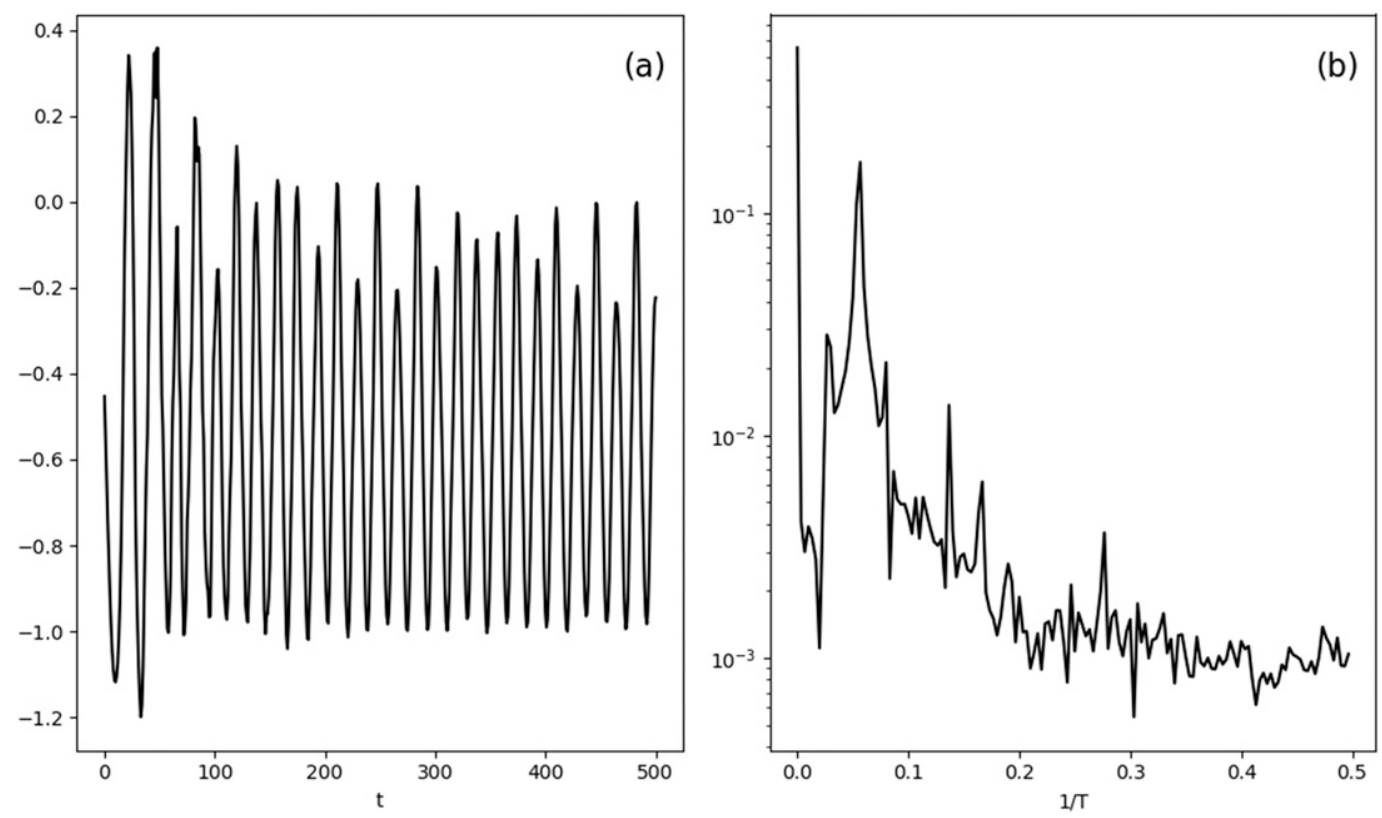

FIG. 6. (a) The dependence on time of a streamfunction value obtained from numerical simulation at the fixed point $(1.2 \pi, 0.6 \pi)$. (b) The corresponding frequency spectrum at time interval $200 \leq t \leq 500$.

Fortunately, in our case this harmonic plays a secondary role and does not impede stable nonlinear oscillations, but instead participates in them. In accord with theory, the amplitudes of $F_{1}$ and $F_{2}$ corresponding to the coefficients $a(t)$ and $\tilde{b}(t)$ oscillate around zero at the same frequency. The amplitude of $F_{3}$ corresponding to $c(t)$ oscillates at large times near a nonzero positive average value. This behavior is consistent with the dependences presented in Fig. 3.

Let us dwell upon the estimates of the vacillation period. According to theory, this period is $T=53.7$ for $c_{0}=0.5$ and $k=0.5$. In the numerical calculations
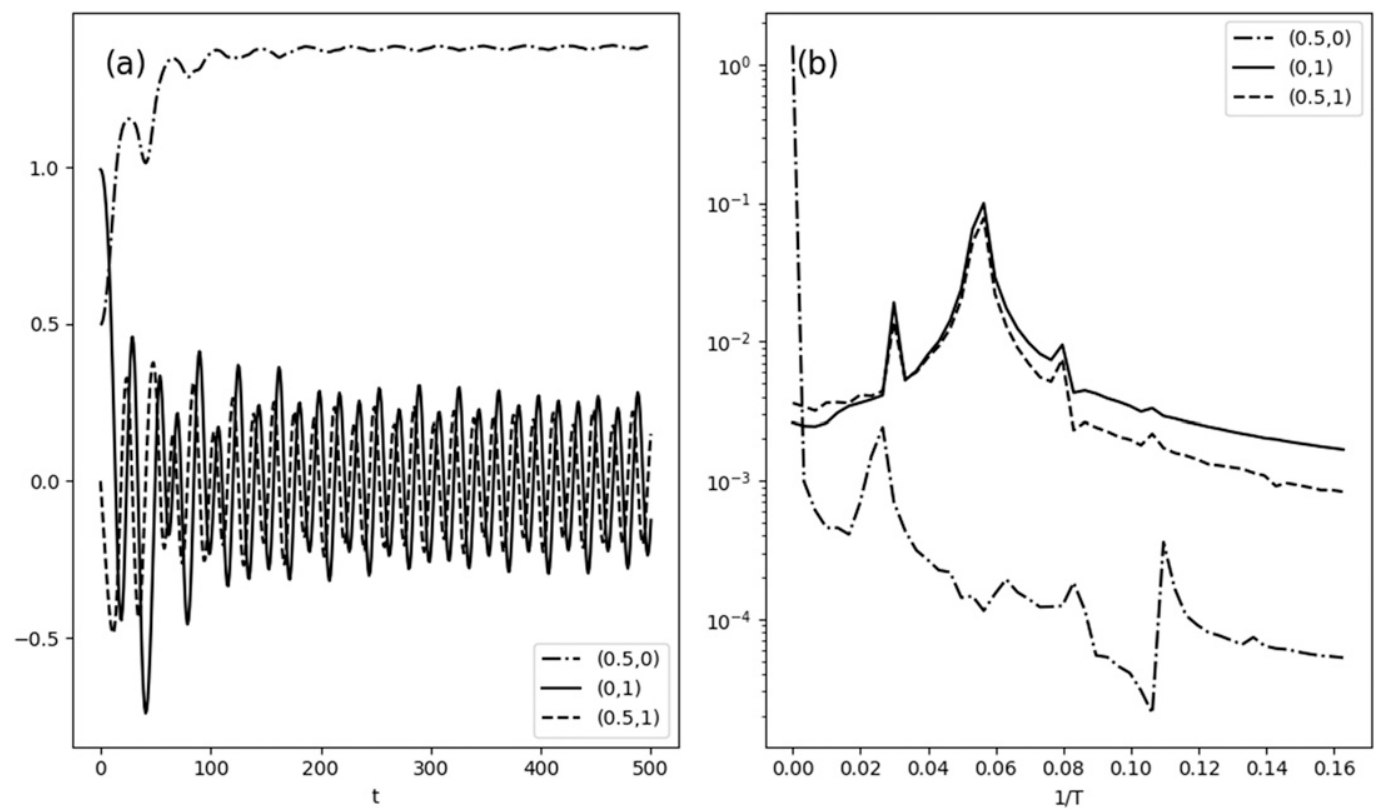

FIG. 7. (a) The dependence on time of the real part of the amplitude of principal large-scale Fourier components of the streamfunction obtained from numerical simulation. (b) The corresponding frequency spectra at time interval $200 \leq t \leq 500$. 
presented in Fig. 6, the period $T \sim 20$ is less than the theoretical value by more than half. In our opinion, this discrepancy may be due to the following reasons. First, a theoretical solution was obtained in the framework of a strongly truncated model. As shown in the next section, in a SQG model with two boundaries, such truncation reduces the increment of the growth of perturbations, compared with the exact value, by a factor of $\sqrt{2}$ (i.e., about $40 \%$ ). The second, more important reason is that for solutions obtained using a numerical code, the fundamental law of conservation of surface potential energy $V$ in the SQG model is not satisfied. As calculations showed, the energy $V$ normalized to the initial value decreases from 1 to 0.6 and only then stabilizes. If we consider that the occurrence of vacillations is due to the conservation of energy, either exact for conservative systems or on average over the period of vacillations for dissipative/forced systems, this drawback of numerical solutions becomes very significant. It must be borne in mind when working with spectral numerical codes.

\section{Instability of a periodic flow in the atmospheric layer of finite height}

We now consider the motion of a stratified rotating atmosphere of finite height $H$. This motion is also described by the dimensionless transport equation for the quasigeostrophic potential vorticity, (1), but with a different choice of characteristic spatial scales. The vertical scale is now taken as $H$, and the horizontal scale is the baroclinic Rossby deformation radius $D=N H / f$. As before, the time scale is equal to $U_{0} / D$, the streamfunction scale is $\Psi_{0}=U_{0} D$. For motions with identically vanishing quasigeostrophic potential vorticity, (1) is reduced to the Laplace equation, (3). The boundary condition (2) is added to this equation, which is now written at two boundaries, $z=0$ and $z=1$.

The Laplace equation, (3), in the layer $0<z<1$ has a solution

$\bar{\psi}=-\frac{\sinh l z}{l \sinh l} \sin l y, \quad \bar{u}=\frac{\sinh l z}{\sinh l} \cos l y, \quad \bar{\sigma}=-\frac{\cosh l z}{\sinh l} \sin l y$,

describing a spatially periodic zonal flow with a period $2 \pi / l$. The flow velocity at the upper boundary is $\bar{u}=\cos l y$, at the lower boundary $\bar{u}=0$. Passing to the limit $l \rightarrow 0$ in (30), we arrive at the Eady (1949) problem with a constant velocity shear $\bar{\psi}=-z y, \bar{u}=z, \bar{\sigma}=-y$. Note that the streamfunction $\bar{\psi}=-(\cosh l z / l \cosh l) \sin l y$ also corresponds to the periodic flow, which contains the hyperbolic cosine. The linear combination of this streamfunction and (30) gives the general form of a periodic flow. For definiteness, we will further consider the flow in (30).

We study the stability of the flow given by (30) in a linear approximation. Representing $\psi=\bar{\psi}+\psi^{\prime}$ and omitting the prime over small perturbations, we obtain the Laplace equation, (3), with boundary conditions

$$
z=0,1: \quad(\partial / \partial t+\bar{u} \partial / \partial x) \psi_{z}-\bar{u}_{z} \psi_{x}=0 .
$$

Following the Galerkin method, we seek approximate solutions to (3) and (31) in the form

$$
\psi=C(x, z, t)+B(x, z, t) \cos l y,
$$

where the expansion coefficients satisfy the equations

$$
B_{x x}+B_{z z}-l^{2} B=0, \quad C_{x x}+C_{z z}=0,
$$

following from the Laplace equation, (3). Note that the choice of two functions in (32) is sufficient to construct a self-consistent solution. Seeking a solution in the form of (13) with three functions automatically leads to the equality $A=0$. We write the velocity profile (30) as $\bar{u}=h(z) \cos l y, h(z)=\sinh l z / \sinh l$. Substituting the expansion (32) into boundary conditions (31) and using the orthogonality conditions for the basis functions $f_{1}=\cos y$ and $f_{2}=1$, we obtain the equations relating the functions $B$ and $C$ on the boundaries

$$
\begin{gathered}
z=0,1: \quad B_{z t}+h(z) C_{x z}-h^{\prime}(z) C_{x}=0, \\
C_{z t}+(1 / 2) h(z) B_{x z}-(1 / 2) h^{\prime}(z) B_{x}=0,
\end{gathered}
$$

where the prime means differentiation with respect to $z$. The description of the dynamics of small perturbations is thus reduced to the solution of (33) and (34).

Equations (33) have solutions in the form of normal modes:

$$
\begin{aligned}
& B=\left(a_{1} \sinh \mu z+b_{1} \cosh \mu z\right) e^{\mathrm{i} k(x-c t),} \\
& C=\left(a_{0} \sinh k z+b_{0} \cosh k z\right) e^{\mathrm{i} k(x-c t),}
\end{aligned}
$$

where $\mu^{2}=k^{2}+l^{2}$ and $c=c_{r}+i c_{i}$ is the complex phase velocity of perturbations; $a_{0}, a_{1}, b_{0}$, and $b_{1}$ are constants. The flow is unstable if normal modes exist with $c_{i}=\operatorname{Im}(c)>0$. The growth rate of such modes is $\lambda=k c_{i}$.

Substituting (35) into (34) leads to a system of four homogeneous algebraic equations with respect to $a_{0}, b_{0}$ and $a_{1}, b_{1}$. After elimination of $b_{0}$ and $b_{1}$, this system is reduced to a system of two equations for $a_{0}$ and $a_{1}$ :

$$
\begin{aligned}
& -c k a_{0}[\cosh k+G(\mu)]+a_{1}\left[c^{2} m k \mu \sinh k\right. \\
& +(1 / 2) F(\mu)]=0,
\end{aligned}
$$




$$
a_{0}\left[2 c^{2} m k \mu \sinh \mu+F(k)\right]-c \mu a_{1}[\cosh \mu+G(k)]=0 .
$$

Here, the notation $m=\sinh l / l$ and

$$
\begin{aligned}
& F(z)=\sinh z(z \operatorname{coth} z-l \operatorname{coth} l), \\
& G(z)=l^{-1}(z \sinh l \sinh z-l \cosh l \cosh z),
\end{aligned}
$$

are used. From the equality to zero of the determinant of the system (36a) and (36b), a quadratic equation follows for $Z=k \mu c^{2}$ :

$$
m^{2} \sinh k \sinh \mu Z^{2}-(1 / 2) P Z+(1 / 4) F(\mu) F(k)=0,
$$

where $P=\cosh k \cosh \mu-2 \cosh l+G(\mu) G(k)$. This equation determines the dependence of the phase velocity $c$ on the perturbation wavenumber $k$ and the main-flow spatial period $2 \pi / l$.

Analysis of (38) shows that it always has two real roots for $l>0$. In this case, the roots can either be both positive or have different signs. The latter case means instability; the imaginary value $c=i(k \mu)^{-1} \sqrt{|Z|}, \operatorname{Im}(c)>0$ corresponds to the negative root $Z$. The case of roots of different signs takes place if the absolute term (1/4) $F(\mu) F(k)$ of (38) is negative. Using expression (37), it is verified that the function $F(z)$ is monotonically increasing. This follows from the observation that $F(z)$ is the product of two monotonically increasing functions, $f(z)=\sinh z$ and $g(z)=z \operatorname{coth} z-l \operatorname{coth} l$. The property in question is evident for $f(z)$ and follows for $g(z)$ from the inequality

$$
\begin{aligned}
\frac{d}{d z} g(z) & =\operatorname{coth} z-\frac{z}{\sinh ^{2} z}=\frac{\cosh z \sinh z-z}{\sinh ^{2} z} \\
& =\frac{\sinh (2 z)-2 z}{2 \sinh ^{2} z}>0 .
\end{aligned}
$$

Since $\mu=\left(k^{2}+l^{2}\right)^{1 / 2}>l$ and $F(l)=0$, then it follows that $F(\mu)>0$; as well as $F(k)>0$ at $k>l$ and $F(k)<0$ at $k<l$. Thus, at a fixed $l$ value, instability occurs for wavenumbers in the interval $0<k<l$.

In view of (38), the expression for the imaginary phase velocity at $0<k<l$ can be written as

$$
\begin{aligned}
& c=i c_{i}=i \frac{1}{\sqrt{k \mu}} \sqrt{\frac{-F(\mu) F(k)}{P+\sqrt{D}}}, \\
& D=P^{2}-4 m^{2} \sinh \mu \sinh k F(\mu) F(k) .
\end{aligned}
$$

The dependences of the growth rate $\lambda=k c_{i}$ on the wavenumber constructed using (39) are shown in Fig. 8. Selected are values $l=1$ and $l=0.8$. As can be seen,

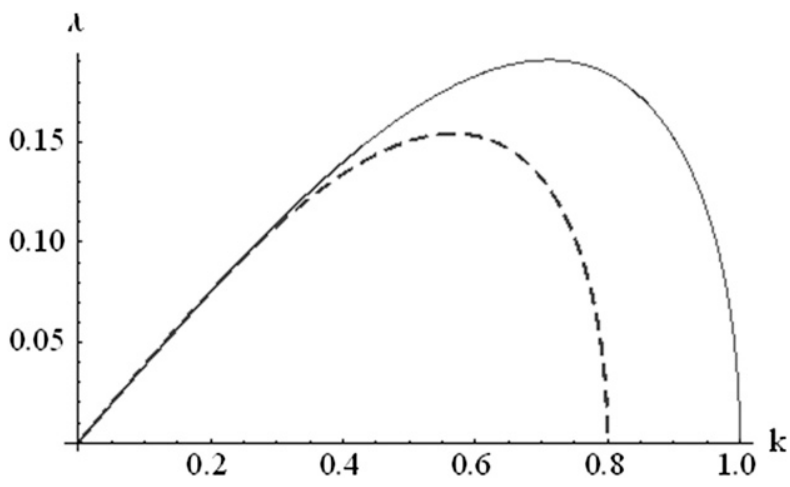

FIG. 8. The dependence of the instability increment $\lambda$ on the wavenumber $k$ for $l=1$ (solid line) and $l=0.8$ (dashed line).

there is long-wave instability with a preferred wavenumber $k$ lying within the interval $0<k<l$.

Above, we have considered wavenumber values $l>0$. A case of $l=0$ corresponding to the Eady (1949) problem with $\bar{u}=z$ requires special consideration. Given that $\mu \rightarrow$ $k, m=\sinh (l) / l \rightarrow 1$, and $l \operatorname{coth} l \rightarrow 1$ at $l \rightarrow 0$, we obtain from (36) the limiting system

$$
\alpha a_{0}+\beta a_{1}=0, \quad 2 \beta a_{0}+\alpha a_{1}=0,
$$

for coefficients $a_{0}$ and $a_{1}$. Here,

$\alpha=-c k^{2} \sinh k, \quad \beta=c k^{2} \sinh k+(1 / 2)(k \cosh k-\sinh k)$.

The condition of solvability of homogeneous system (40) leads to a quadratic equation for the complex phase velocity:

$$
c^{2}-\frac{1}{\sqrt{2}} c+\frac{1}{2}\left(k^{-1} \operatorname{coth} k-k^{-2}\right)=0 .
$$

Equation (41) can be compared with the exact equation arising in the linear two-dimensional Eady model (Pedlosky 1987):

$$
c^{2}-c+\left(k^{-1} \operatorname{coth} k-k^{-2}\right)=0 .
$$

From (41) and (42) it follows, respectively,

$$
\begin{aligned}
c & =\frac{1}{2 \sqrt{2}} \pm \frac{1}{k \sqrt{2}} \sqrt{D}, \quad c=\frac{1}{2} \pm \frac{1}{k} \sqrt{D}, \\
D & =\left(k^{2} / 4\right)-k \operatorname{coth} k+1 .
\end{aligned}
$$

Given an identity $D=[k / 2-\operatorname{coth}(k / 2)][k / 2-\tanh (k / 2)]$, the discriminant $D<0$ within the interval $0<k<k_{b}$ where $k_{b}=2.399$ is the root of the equation $\operatorname{coth}(k / 2)=$ $k / 2$. Direct comparison of two expressions for $c$ in (43) shows that the Galerkin method with two basis functions (i) leads to an accurate determination of the 
interval of unstable wavenumbers, (ii) gives the exact value for the wavenumber of the fastest growing mode $\left(k_{m}=1.606\right)$, (iii) decreases the instability increment value $\lambda=k c_{i}$ in $\sqrt{2}$ times (about $40 \%$ ), and (iv) reduces the phase propagation velocity of a mode by a factor of $\sqrt{2}$. Given the presence of only two basis functions, the indicated errors, in our opinion, are permissible for the purposes of a qualitative analysis.

Concluding this section, we note that using the Galerkin method for flow in a layer, a nonlinear model of baroclinic instability can also be constructed, describing the occurrence of continuous oscillations in the flow.

\section{Concluding remarks}

Extensive literature is devoted to the stability study of spatially periodic flows of geophysical fluids. Existing research mainly focuses on the two-dimensional Kolmogorov flow and its analog in a stratified fluid (see, e.g., Kalashnik and Kurgansky 2018; Balmforth and Young 2002 , and references therein). Separate works were devoted to the stability of spatially periodic flows in the form of a monochromatic Rossby wave (Lorenz 1972; Dolzhansky 1985, 2013) and/or a monochromatic internal gravity wave (Mied 1976; Drazin 1977; Kurgansky 1979, 1980; see also Kurgansky 2018, and references therein). This paper presents the results of stability study of spatially periodic flows in the framework of the SQG equations (Blumen 1978; Held et al. 1995). The corresponding model has been developed in this work to describe the dynamics of geophysical fluid flows with vanishing quasigeostrophic potential vorticity in the presence of one or two horizontal boundaries. In the meteorological context, such boundaries are the underlying Earth surface and the tropopause.

To study the linear stability of a spatially periodic flow with one boundary (underlying surface), we used two approaches. In the first approach, the solution of the linear stability problem is sought by decomposing into a trigonometric series, and the growth rate of the perturbations is found from the characteristic equation containing an infinite continued fraction. Truncating the continued fraction leads to successive approximations for the increment of instability. In the second approach, few-mode Galerkin approximations of the solution are constructed. From the conditions of orthogonality to basis trigonometric functions, a linear system of ordinary differential equations is formulated, which makes it possible to find an increment of instability. As shown in the paper, both approaches lead to the same dependence of the growth increment on the wavenumber. The existence of instability with a preferred horizontal scale on the order of the wavelength of the main flow follows from this dependence. A similar result was obtained in the framework of the SQG model with two horizontal surfaces. A spatially periodic flow localized near the upper boundary (tropopause) is unstable with respect to perturbations with a wavelength greater than the wavelength of the main flow.

Using the Galerkin method, we also investigated the features of the nonlinear dynamics of flow perturbations with the lower boundary solely. A nonlinear version of the Galerkin system of equations is formulated, analogous to the system of equations describing the dynamics of a symmetric top in classical mechanics (Landau and Lifshitz 1982) or the fluid motion in an ellipsoidal cavity (cf. Gledzer et al. 1981). It is shown that the exponential growth of disturbances at the linear stage is always replaced by a stage of stable nonlinear oscillations (vacillations). These oscillations are similar to nonlinear oscillations arising from the loss of stability of a geostrophic flow with a velocity jump in a two-layer fluid (Pedlosky 1970, 1987; Barcilon and Drazin 1984). An analytical formula for the oscillation period, determining its dependence on the amplitude of the initial perturbation, is obtained. It is shown that the process of formation of closed vortex structures, which is periodic in time, is associated with these oscillations. The structures grow during the first half of the time period and completely disappear at the end of the period. Numerical integration of the full nonlinear SQG equations with initial conditions that fit the theoretical considerations confirmed the formation at large times of a regime of stable nonlinear oscillations.

We note that a number of important issues remained outside the scope of the work. The presented model of nonlinear dynamics actually describes the "unstable wave-main flow" interaction process. As well as in the theory by Pedlosky and Polvani (1987), it is of interest to additionally explore the "wave-wave" interaction process. Another important issue is the study of stability with account for dissipative factors. It is this question related to the determination of the critical value of the Reynolds number that stimulated the first studies of the Kolmogorov flow stability. One possible variant of the stability problem with account for the momentum and buoyancy horizontal diffusion is discussed in appendix A. It provides additional justification for the validity of Galerkin procedure applied in the paper.

In conclusion, it should be emphasized that the stability of model periodic flows with a sinusoidal velocity profile was investigated in this work. The periodic systems of large-scale jets in the Jovian atmosphere or the multiple jets and fronts found in the Southern Ocean are 
geophysical prototypes of such flows. As observational data show, the instability of multiple jets in the Antarctic Circumpolar Current manifests itself in their meandering, merging and diverging, and changes in intensity. For a quantitative comparison with theory, it is necessary to conduct carefully controlled laboratory experiments similar to the classical experiments by Hide (1953), where for the first time a nonlinear oscillation regime was found that is close to that described in this work.

Acknowledgments. The work was supported by the Russian Foundation for Basic Research (Project 1805-00414; sections 1-3 and appendix A) and the Russian Science Foundation (Project 19-17-00248; sections 4-6 and appendix B). We thank Pavel Perezhogin for helpful discussions on the material in section 6 . We are also grateful to three anonymous reviewers whose critical comments and useful suggestions helped greatly to improve the content and presentation of material of this manuscript.

\section{APPENDIX A}

\section{Stability Analysis with Account for Dissipative Factors}

In the presence of the buoyancy source and also the horizontal diffusion of the momentum and buoyancy with coefficients $\nu$ and $\chi$, accordingly, such that the Prandtl number, $\operatorname{Pr}=\nu / \chi=1,(1)$ and (2) take the form

$$
\begin{aligned}
& q_{t}+J(\psi, q)=\mathrm{Pe}^{-1} \nabla^{2} q+\mathrm{Pe}^{-1} \partial \hat{Q} / \partial z, \\
& q=\nabla^{2} \psi+\psi_{z z} \equiv \psi_{x x}+\psi_{y y}+\psi_{z z}, \\
& z=0: \quad \psi_{z t}+J\left(\psi, \psi_{z}\right)=\mathrm{Pe}^{-1} \nabla^{2} \psi_{z}+\mathrm{Pe}^{-1} \hat{Q} .
\end{aligned}
$$

Here, $\mathrm{Pe}=U_{0} D / \chi$ is the Peclet number, which in this case is equal to the Reynolds number, $\operatorname{Re}=U_{0} D / \nu$, and $\hat{Q}=Q / Q_{0}$ is the nondimensional buoyancy source, where the function $Q$ specifies the spatial distribution of the buoyancy source and is nondimensionalized using the scale $Q_{0}=U_{0} N \chi / D^{2}$.

We take $\hat{Q}=-\exp (-\varepsilon z) \cos y$ in (A1) and (A2), where $\varepsilon \ll 1$. Then, asymptotically in $\varepsilon$ we arrive at the problem

$$
\begin{aligned}
q_{t}+J(\psi, q) & =\mathrm{Pe}^{-1} \nabla^{2} q, \\
q & =\nabla^{2} \psi+\psi_{z z} \equiv \psi_{x x}+\psi_{y y}+\psi_{z z}, \\
z=0: \quad \psi_{z t} & +J\left(\psi, \psi_{z}\right)=\mathrm{Pe}^{-1}\left(\nabla^{2} \psi_{z}-\cos y\right) .
\end{aligned}
$$

In this case, the following energy balance equations hold, comparing (4a) and (4b),

$$
\begin{aligned}
& \frac{d E}{d t}=-\mathrm{Pe}^{-1} \int_{0}^{\infty} \overline{\left[\left(\nabla^{2} \psi\right)^{2}+\left(\nabla \psi_{z}\right)^{2}\right]} d z+\mathrm{Pe}^{-1} \overline{\psi \cos y}, \\
& \frac{d}{d t} V=-\mathrm{Pe}^{-1} \overline{\left(\nabla \psi_{z}\right)^{2}}-\mathrm{Pe}^{-1} \overline{\psi_{z} \cos y}
\end{aligned}
$$

that is, (A3) and (A4) represent a well-posed problem with nonnegative dissipative functions defined by (A5) and (A6).

Again, as in section 2, the class of flows described by solutions to the Laplace equation, (3), which identically satisfy (A3), is considered. The exact stationary solution of (A3) and (A4) is identical to (5). Accordingly, we obtain the equation of the linear theory of stability:

$$
z=0: \quad \psi_{z t}+\sin y\left(\psi_{x z}+\psi_{x}\right)=\operatorname{Pe}^{-1} \nabla^{2} \psi_{z} .
$$

The solution of this equation by the method specified in section 3 leads to (9), where now $\tilde{a}_{n}=$ $(2 / k)\left(\lambda+k_{n}^{2} / \mathrm{Pe}\right)\left[k_{n} /\left(k_{n}-1\right)\right]$. The characteristic equation to determine the increment $\lambda$ has again the form (10). By analogy with Meshalkin and Sinai (1961), the positive values of the real part of $\lambda$, which provide instability, exist only for $k<1$. Moreover, $\lambda$ values that have a nonnegative real part are always real; that is, the so-called principle of exchange of stabilities holds (Chandrasekhar 1981). The instability estimate follows from the inequalities $\left[0 ; \tilde{a}_{1}\right]<\left[0 ; \tilde{a}_{1}, \tilde{a}_{2}, \ldots\right]<\left[0 ; \tilde{a}_{1}, \tilde{a}_{2}\right]$ and replacing the infinite continued fraction with its convergent $\left[0 ; \tilde{a}_{1}\right]$ we obtain the characteristic equation

$$
\lambda^{2}+\left(k^{2}+k_{1}^{2}\right) \mathrm{Pe}^{-1} \lambda+k^{2} k_{1}^{2}\left(\mathrm{Pe}^{-2}-\mathrm{Pe}_{\text {crit }}^{-2}\right)=0,
$$

where the critical value of the Peclet number as a function of $k$,

$$
\mathrm{Pe}_{\text {crit }}^{2}=\frac{2 k k_{1}^{3}}{(1-k)\left(k_{1}-1\right)},
$$

describes the neutral stability curve, owing to the principle of exchange of stabilities. A remarkable difference from the Kolmogorov flow, where the instability is long wave, that is, occurs at $k^{2} \rightarrow 0$, and minimum critical Reynolds number, $\operatorname{Re}=\sqrt{2}$, is that, in our case, the instability occurs at $k$ values approximately in the middle of the interval $(0,1)$ and the minimum critical Peclet number exceeds $\sqrt{2}$; see Fig. A1. For Pe $\gg \mathrm{Pe}_{\text {crit }}$, (A7) has the solution

$$
\lambda_{1,2}= \pm \frac{k k_{1}}{\mathrm{Pe}_{\text {crit }}}-\frac{k^{2}+1 / 2}{\mathrm{Pe}}+O\left(\mathrm{Pe}^{-2}\right),
$$




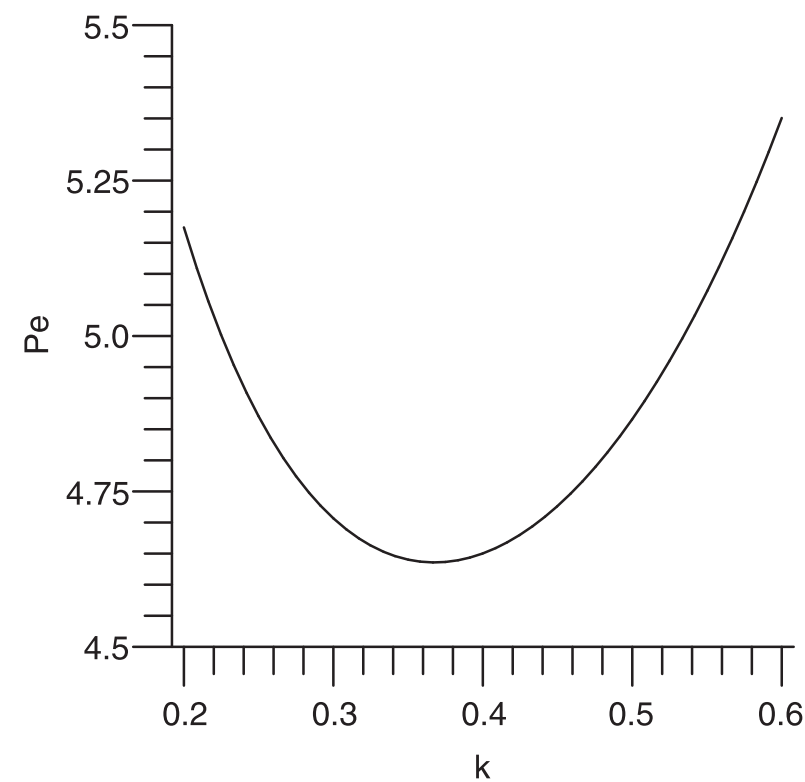

FIG. A1. The dependence of critical values of the Peclet number Pe on the wavenumber $k$ near the minimum of the neutral stability curve for a problem with the horizontal diffusion of momentum and buoyancy for Prandtl number $\operatorname{Pr}=1$.

which shows that (11) provides a correct expression for the instability increment for very large Peclet numbers.

Returning to the question of determining the solution $\left\{d_{n}\right\}$ of (9), we have for large $n>0$ from (9) that

$$
\frac{d_{n-1}}{d_{n}}=-\tilde{a}_{n}+\frac{1}{d_{n} / d_{n+1}} \approx-\tilde{a}_{n} \approx-\frac{2 k_{n}^{3}}{\operatorname{Pe} k\left(k_{n}-1\right)} \approx-\frac{2 n^{2}}{\operatorname{Pe} k} .
$$

As a consequence, the solution to (9) has an asymptotic behavior $d_{n} \propto(-1)^{n}(n !)^{-2}, n>0$. Similar arguments can be applied for $n<0$. Note that this asymptotics is very "deep," since it starts working well already from $n=3$. Approximating the right-hand side of $(10)$ with $\left[0 ; a_{1}\right]$ is equivalent to restricting with three components $\left\{d_{-1}, d_{0}\right.$, $d_{1}$ \}, whereas all other $d_{n}$ are set equal to zero. The same result holds for a restriction with five nonzero components $\left\{d_{-2}, d_{-1}, d_{0}, d_{1}, d_{2}\right\}$ that corresponds to (12). This proves that the closeness of $\lambda$ values between the first approximation to the solution, $\left\{d_{-1}, d_{0}, d_{1}\right\}$, and the exact solution, $\left\{d_{n}\right\}$, translates into closeness of the solutions themselves, only if a very small but finite momentum and buoyancy diffusivity is introduced.

For large-scale motions in the atmosphere, the Peclet number is very large and an approximate approach based on (11) can be used. Hereby, justification of this approach was provided. Taking into account the Peclet number finiteness is irrelevant when formally approximating an infinite continued fraction in (10) with it convergent of the lowest order, but is necessary to estimate the accuracy of the corresponding approximation of the exact solution $\left\{d_{\mathrm{n}}\right\}$ of (9) when applying (11).

\section{APPENDIX B}

\section{Galerkin Approximation with Five Basis Functions}

We seek a solution to the linearized problem (3) and (6) in the form of an expansion

$$
\begin{aligned}
\psi= & A^{(1)}(x, z, t) \sin y+B^{(1)}(x, z, t) \cos y+C(x, z, t) \\
& +A^{(2)}(x, z, t) \sin (2 y)+B^{(2)}(x, z, t) \cos (2 y),
\end{aligned}
$$

additionally taking into account trigonometric functions of double argument. From the Laplace equation, (3), we obtain the equations for the expansion coefficients:

$$
\begin{array}{r}
C_{x x}+C_{z z}=0, \quad A_{x x}^{(1)}+A_{z z}^{(1)}-A^{(1)}=0, \\
A_{x x}^{(2)}+A_{z z}^{(2)}-4 A^{(2)}=0 .
\end{array}
$$

Functions $B^{(1)}$ and $B^{(2)}$ satisfy the same equations as $A^{(1)}$ and $A^{(2)}$ do.

Equations on the boundary $z=0$ are added to (B2). They are obtained by substituting (B1) into the linearized boundary condition (6). From the conditions of orthogonality to five basis functions $1, \sin y, \cos y, \sin (2 y)$, and $\cos (2 y)$, there follows a system of five boundary equations, which splits into two closed subsystems. The first subsystem takes the form

$$
\begin{aligned}
C_{z t}+(1 / 2)\left(A_{x z}^{(1)}+A_{x}^{(1)}\right) & =0, \\
A_{z t}^{(1)}+C_{x z}+C_{x}-(1 / 2)\left(B_{x z}^{(2)}+B_{x}^{(2)}\right) & =0, \\
B_{z t}^{(2)}-(1 / 2)\left(A_{x z}^{(1)}+A_{x}^{(1)}\right) & =0 .
\end{aligned}
$$

The second subsystem reads

$$
\begin{aligned}
& B_{z t}^{(1)}+(1 / 2)\left(A_{x z}^{(2)}+A_{x}^{(2)}\right)=0, \\
& A_{z t}^{(2)}+(1 / 2)\left(B_{x z}^{(1)}+A_{x}^{(1)}\right)=0 .
\end{aligned}
$$

We emphasize that (B3) and (B4) are written for the boundary $z=0$.

Searching for a solution of (B2) in the form

$$
\begin{aligned}
C & =c(t) e^{-k z} \cos (k x), \quad A^{(1)}=a^{(1)}(t) e^{-k_{1} z} \sin (k x), \\
B^{(2)} & =b^{(2)}(t) e^{-k_{2} z} \cos (k x),
\end{aligned}
$$

where $k_{n}=\sqrt{k^{2}+n^{2}}$, we obtain from (B3) the system of ordinary differential equations with respect to $c(t)$, $a^{(1)}(t)$, and $b^{(2)}(t)$. This system is reduced to one equation: 


$$
a_{t t}^{(1)}-\lambda^{2} a^{(1)}=0, \quad \lambda^{2}=\frac{\left(k_{1}-1\right) k\left[2 k_{2}-\left(3 k_{2}-1\right) k\right]}{4 k_{1} k_{2}},
$$

which describes the instability with increment (12), corresponding to the second approximation of the theory of continued fractions. Thus, the second approximation (12) can be obtained by the Galerkin method with account for trigonometric basis functions of double argument.

For solutions of (B2) of the form

$$
A^{(2)}=a^{(2)}(t) e^{-k_{2} z} \sin (k x), \quad B^{(1)}=b^{(1)}(t) e^{-k_{1} z} \cos (k x),
$$

a system of differential equations with respect to $a^{(2)}(t)$ and $b^{(1)}(t)$ follows from subsystem (B4), which reduces to

$$
a_{t t}^{(2)}+\omega^{2} a^{(2)}=0, \quad \omega^{2}=\frac{k^{2}\left(k_{1}-1\right)\left(k_{2}-1\right)}{4 k_{1} k_{2}} .
$$

Equation (B6) describes stable oscillations with frequency $\omega$.

\section{REFERENCES}

Abramowitz, M., and I. A. Stegun, Eds., 1964: Handbook of Mathematical Functions, Graphs and Mathematical Tables. Applied Mathematics Series, Vol. 55, U.S. Government Printing Office, $1046 \mathrm{pp}$.

Arbic, B. K., and G. R. Flierl, 2003: Coherent vortices and kinetic energy ribbons in asymptotic, quasi two-dimensional f-plane turbulence. Phys. Fluids, 15, 2177-2189, https://doi.org/ 10.1063/1.1582183.

Badin, G., 2013: Surface semi-geostrophic dynamics in the ocean. Geophys. Astrophys. Fluid Dyn., 107, 526-540, https://doi.org/ 10.1080/03091929.2012.740479.

_ 2014: On the role of non-uniform stratification and shortwave instabilities in three-layer quasi-geostrophic turbulence. Phys. Fluids, 26, 096603, https://doi.org/10.1063/ 1.4895590

— and A. M. Barry, 2018: Collapse of generalized Euler and surface quasigeostrophic point vortices. Phys. Rev., 98E, 023110, https://doi.org/10.1103/PhysRevE.98.023110.

_ , and F. Poulin, 2018: Asymptotic scale-dependent stability of surface quasi-geostrophic vortices: Semi-analytic results. $G e$ ophys. Astrophys. Fluid Dyn., 113, 574-593, https://doi.org/ 10.1080/03091929.2018.1453930.

Balmforth, N. J., and Y.-N. Young, 2002: Stratified Kolmogorov flow. J. Fluid Mech., 450, 131-167, https://doi.org/10.1017/ S0022111002006371.

Barcilon, A., and P. G. Drazin, 1984: A weakly nonlinear theory of amplitude vacillation and baroclinic waves. J. Atmos. Sci., 41, 3314-3330, https://doi.org/10.1175/1520-0469(1984)041<3314: AWNTOA $>2.0 . \mathrm{CO} ; 2$.

Berloff, P., I. Kamenkovich, and J. Pedlosky, 2009: A mechanism of formation of multiple zonal jets in the oceans. J. Fluid Mech., 628, 395-425, https://doi.org/10.1017/S0022112009006375.

Blumen, W., 1978: Uniform potential vorticity flow. Part I: Theory of wave interactions and two-dimensional turbulence. J. Atmos.
Sci., 35, 774-783, https://doi.org/10.1175/1520-0469(1978) 035<0774:UPVFPI $>2.0$. CO;2.

Buzyna, G., R. L. Pfeffer, and R. Kung, 1989: Kinematic properties of wave amplitude vacillation in a thermally driven rotating fluid. J. Atmos. Sci., 46, 2716-2729, https://doi.org/10.1175/ 1520-0469(1989)046<2716:KPOWAV>2.0.CO;2.

Canuto, C., M. Y. Hussaini, A. Quarteroni, and T. A. Zang, 1988: Spectral Methods in Fluid Dynamics. Springer Verlag, 567 pp.

Capet, X., P. Klein, B. Hua, G. Lapeyre, and J. McWilliams, 2008: Surface kinetic energy transfer in surface quasi-geostrophic flows. J. Fluid Mech., 604, 165-174, https://doi.org/10.1017/ S0022112008001110.

Carton, X., 2009: Instability of surface quasigeostrophic vortices. J. Atmos. Sci., 66, 1051-1062, https://doi.org/10.1175/ 2008JAS2872.1.

_- D. Ciani, J. Verron, J. Reinaud, and M. Sokolovskiy, 2016: Vortex merger in surface quasigeostrophy. Geophys. Astrophys. Fluid Dyn., 110, 1-22, https://doi.org/10.1080/ 03091929.2015.1120865.

Chandrasekhar, S., 1981: Hydrodynamic and Hydromagnetic Stability. Dover Publications, 654 pp.

Constantin, P., A. J. Majda, and E. G. Tabak, 1994: Singular front formation in a model of quasigeostrophic flow. Phys. Fluids, $\mathbf{6}$, 9-11, https://doi.org/10.1063/1.868050.

Conti, G., and G. Badin, 2019: Velocity statistics for point vortices in the local $\alpha$-models of turbulence. Geophys. Astrophys. Fluid Dyn., 113, 527-552, https://doi.org/10.1080/03091929.2019.1572750.

Dolzhansky, F. V., 1985: Effect of Ekman layer on the stability of planetary waves. Izv. Atmos. Ocean. Phys., 21, 292-297.

- 2013: Fundamentals of Geophysical Hydrodynamics. Springer-Verlag, $272 \mathrm{pp}$.

Drazin, P. G., 1977: On the instability of an internal gravity wave Proc. Roy. Soc. London, 356A, 411-432, https://doi.org/ 10.1098/rspa.1977.0142.

Dritschel, D., 2011: An exact steadily rotating surface quasigeostrophic elliptical vortex. Geophys. Astrophys. Fluid Dyn., 105, 368-376, https://doi.org/10.1080/03091929.2010.485997.

Eady, E. T., 1949: Long waves and cyclone waves. Tellus, 1 (3), $33-$ 52, https://doi.org/10.3402/tellusa.v1i3.8507.

Galperin, B., and P. L. Read, Eds., 2019: Zonal Jets: Phenomenology, Genesis, and Physics. Cambridge University Press, 504 pp., https://doi.org/10.1017/9781107358225.

Gledzer, E. B., F. V. Dolzhansky, and A. M. Obukhov, 1981: Systems of Hydrodynamic Type and Their Applications (in Russian). Nauka, 366 pp.

Hakim, G. J., C. Snyder, and D. J. Muraki, 2002: A new surface model for cyclone-anticyclone asymmetry. J. Atmos. Sci., 59 2405-2420, https://doi.org/10.1175/1520-0469(2002)059<2405: ANSMFC $>2.0 . \mathrm{CO} ; 2$.

Harvey, B., and M. Ambaum, 2010: Instability of surfacetemperature filaments in strain and shear. Quart. J. Roy. Meteor. Soc., 136, 1506-1513, https://doi.org/10.1002/ qj.651. , and _- 2011: Perturbed Rankine vortices in surface quasigeostrophic dynamics. Geophys. Astrophys. Fluid Dyn., 105, 377-391, https://doi.org/10.1080/03091921003694719.

,$- \ldots$, and X. Carton, 2011: Instability of shielded surface temperature vortices. J. Atmos. Sci., 68, 964-971, https:// doi.org/10.1175/2010JAS3669.1.

Held, I. M., R. T. Pierrehumbert, S. T. Garner, and K. L. Swanson, 1995: Surface quasigeostrophic dynamics. J. Fluid Mech., 282, 1-20, https://doi.org/10.1017/S0022112095000012. 
Hide, R., 1953: Some experiments on thermal convection in a rotating liquid. Quart. J. Roy. Meteor. Soc., 79, 161-161, https:// doi.org/10.1002/qj.49707933916.

Juckes, M., 1994: Quasigeostrophic dynamics of the tropopause. J. Atmos. Sci., 51, 2756-2768, https://doi.org/10.1175/15200469(1994)051<2756:QDOTT>2.0.CO;2.

Kalashnik, M., and M. Kurgansky, 2018: Nonlinear dynamics of long-wave perturbations of the Kolmogorov flow for large Reynolds numbers. Ocean Dyn., 68, 1001-1012, https:// doi.org/10.1007/s10236-018-1180-6.

Khinchin, A. Ya., 1964: Continued Fractions. University of Chicago Press, 95 pp.

Kim, S., and H. Okamoto, 2015: Unimodal patterns appearing in the Kolmogorov flows at large Reynolds numbers. Nonlinearity, $\mathbf{2 8}$, 3219-3242, https://doi.org/10.1088/0951-7715/28/9/3219.

Kurgansky, M. V., 1979: Hydrodynamic instability of internal waves in the atmosphere. Izv. Atmos. Ocean. Phys., 15, 707-710.

_ 1980: Instability of internal gravity waves propagating at small angles to the vertical. Izv. Atmos. Ocean. Phys., 16, 758764.

_- 2018: On the instability of internal gravity waves propagating at small but finite angles to the vertical. Geophys. Astrophys. Fluid Dyn., 112, 265-276, https://doi.org/ 10.1080/03091929.2018.1510920.

Kuznetsov, A. P., S. P. Kuznetsov, and N. M. Ryskin, 2002: Nonlinear Oscillations (in Russian). Fizmatlit, 292 pp.

Landau, L. D., and E. M. Lifshitz, 1982: Mechanics. 3rd ed. Course of Theoretical Physics, Vol. 1, Butterworth-Heinemann, 224 pp.

Lapeyre, G., and P. Klein, 2006: Dynamics of the upper oceanic layers in terms of surface quasigeostrophy theory. J. Phys. Oceanogr., 36, 165-176, https://doi.org/10.1175/JPO2840.1.

Lim, C., and A. Majda, 2001: Point vortex dynamics for coupled surface/interior QG and propagating heton clusters in models for ocean convection. Geophys. Astrophys. Fluid Dyn., 94, 177-220, https://doi.org/10.1080/03091920108203407.

Lorenz, E. N., 1972: Barotropic instability of Rossby wave motion. J. Atmos. Sci., 29, 258-264, https://doi.org/10.1175/15200469(1972)029<0258:BIORWM > 2.0.CO;2.

Lucas, D., and R. Kerswell, 2014: Spatiotemporal dynamics in twodimensional Kolmogorov flow over large domains. J. Fluid Mech., 750, 518-554, https://doi.org/10.1017/jfm.2014.270.

Maximenko, N. A., B. Bang, and H. Sasaki, 2005: Observational evidence of alternating jets in the World Ocean. Geophys. Res. Lett., 32, L12607, https://doi.org/10.1029/2005GL022728.

McWilliams, J. C., 2016: Submesoscale currents in the ocean. Proc. Roy. Soc., 472A, 20160117, https://doi.org/10.1098/ rspa.2016.0117.

Merilees, P. E., 1972: On the periods of amplitude vacillations. J. Meteor. Soc. Japan, 50, 214-225, https://doi.org/10.2151/ jmsj1965.50.3_214.

Meshalkin, L. D., and Y. G. Sinai, 1961: Investigation of the stability of a stationary of equations for the plane movement of an incompressible viscous fluid. J. Appl. Math. Mech., 25, 1700-1705, https://doi.org/10.1016/0021-8928(62)90149-1.

Mied, R. P., 1976: The occurrence of parametric instabilities in finite-amplitude internal gravity waves. J. Fluid Mech., 78, 763-784, https://doi.org/10.1017/S0022112076002735.

Muraki, D. J., and C. Snyder, 2007: Vortex dipoles for surface quasigeostrophic models. J. Atmos. Sci., 64, 2961-2967, https:// doi.org/10.1175/JAS3958.1.

Ohkitani, K., and T. Sakajo, 2010: Oscillatory damping in long-time evolution of the surface quasi-geostrophic equations with generalized viscosity: A numerical study. Nonlinearity, 23, 30293051, https://doi.org/10.1088/0951-7715/23/12/003; Corrigendum, 25, 2181-2202, https://doi.org/10.1088/0951-7715/25/7/2181

Pedlosky, J., 1970: Finite amplitude baroclinic waves. J. Atmos. Sci., 27, 15-30, https://doi.org/10.1175/1520-0469(1970) $027<0015$ :FABW $>2.0 . \mathrm{CO} ; 2$.

1987: Geophysical Fluid Dynamics. Springer-Verlag, 710 pp. , and L. Polvani, 1987: Wave-wave interaction of unstable baroclinic waves. J. Atmos. Sci., 44, 631-647, https://doi.org/ 10.1175/1520-0469(1987)044<0631:WIOUBW > 2.0.CO;2.

Ragone, F., and G. Badin, 2016: A study of surface semigeostrophic turbulence: Freely decaying dynamics. J. Fluid Mech., 792, 740-774, https://doi.org/10.1017/jfm.2016.116.

Reinaud, J., D. Dritschel, and X. Carton, 2016: Interaction between a surface quasi-geostrophic buoyancy filament and an internal vortex. Geophys. Astrophys. Fluid Dyn., 110, 461-490, https://doi.org/10.1080/03091929.2016.1233331.

——, — , and ——, 2017: Interaction between a surface quasi-geostrophic buoyancy anomaly jet and internal vortices. Phys. Fluids, 29, 086603, https://doi.org/10.1063/ 1.4999474

Sokolov, S., and S. R. Rintoul, 2007: Multiple jets of the Antarctic Circumpolar Current south of Australia. J. Phys. Oceanogr., 37, 1394-1412, https://doi.org/10.1175/JPO3111.1.

Solomon, T. H., W. J. Holloway, and H. L. Swinney, 1993: Shear flow instabilities and Rossby waves in barotropic flow in a rotating annulus. Phys. Fluids, 5A, 1971-1982, https://doi.org/ 10.1063/1.858824.

Tulloch, R., and K. S. Smith, 2009a: Quasigeostrophic turbulence with explicit surface dynamics: Application to the atmospheric energy spectrum. J. Atmos. Sci., 66, 450-467, https://doi.org/ 10.1175/2008JAS2653.1.

, and $-2009 \mathrm{~b}$ : A note on the numerical representation of surface dynamics in quasigeostrophic turbulence: Application of the nonlinear Eady model. J. Atmos. Sci., 66, 1063-1068, https://doi.org/10.1175/2008JAS2921.1.

Vallis, G. K., 2006: Atmospheric and Oceanic Fluid Dynamics: Fundamentals and Large-Scale Circulation. Cambridge University Press, 745 pp.

Vasavada, A., and A. P. Showman, 2005: Jovian atmospheric dynamics: An update after Galileo and Cassini. Rep. Prog. Phys., 68, 1935-1996, https://doi.org/10.1088/0034-4885/68/8/R06. 